\title{
Model reduction for LPV systems based on approximate modal decomposition
}

\author{
T. Luspay, T. Péni*, I. Gőzse, Z. Szabó, B. Vanek \\ Systems and Control Laboratory of Institute for Computer Science and Control, 1111 Budapest, Kende u. 13-17., \\ Hungary
}

\begin{abstract}
SUMMARY
The paper presents a novel model order reduction technique for large-scale linear parameter varying (LPV) systems. The approach is based on decoupling the original dynamics into smaller dimensional LPV subsystems that can be independently reduced by parameter varying reduction methods. The decomposition starts with the construction of a modal transformation that separates the modal subsystems. Hierarchical clustering is applied then to collect the dynamically similar modal subsystems into larger groups. The resulting parameter varying subsystems are then independently reduced. This approach substantially differs from most of the previously proposed LPV model reduction techniques, since it performs manipulations on the LPV model itself, instead of on a set of linear time-invariant (LTI) models defined at fixed scheduling parameter values. Therefore the interpolation, which is often a challenging part in reduction techniques, is inherently solved. The applicability of the developed algorithm is thoroughly investigated and demonstrated by numerical case studies. Copyright (c) 2010 John Wiley \& Sons, Ltd.
\end{abstract}

Received ...

KEY WORDS: linear parameter-varying systems, model order reduction, balanced realization, modal transformation, clustering

\section{INTRODUCTION}

The LPV models have proven to be useful in system analysis and control design, due to their ability to represent a wide class of nonlinear systems, while preserving the advantageous properties of linear structures [1], [2], [3]. Using LPV models, the analysis and control synthesis tasks are generally casted to convex optimization problems involving linear matrix inequality (LMI) constraints [4]. As long as the complexity of the system is low (the dimension of the state is smaller than 20-30, the number of scheduling variables is at most 2 or 3 ) these problems can be efficiently solved by off-the-shelf semidefinite solvers. On the other hand, the modeling of complex systems (e.g. flexible structures [5]) results in high dimensional LPV systems (even with 100-1000 states), the dimension of which has to be reduced in order to make them numerically tractable. It is important to emphasize that our aim is to reduce the dimension of the state vector and not of the scheduling variable. The latter problem is fundamentally different and is addressed in e.g. [6], [7].

Model order reduction for linear time invariant (LTI) systems is a well-studied topic, see e.g. [8] and the references therein. The same problem for LPV systems was first addressed in [9], [10], where the concept of balanced realization based model truncation [11] was extended to parameter varying systems. The approach is based on the balancing state transformation computed from the parametervarying controllability and observability Gramians. These computations involve LMI optimization,

${ }^{*}$ Correspondence to: Tamas Péni, Systems and Control Laboratory of Institute for Computer Science and Control, 1111 Budapest, Kende u. 13-17., Hungary, E-mail: peni .tamas@s ztaki .mta.hu 
which suffers from the same computational limitations as the LPV analysis and synthesis problems. Therefore, this model reduction technique can only be applied to systems of moderate complexity. In order to avoid these limitations an approximate balanced truncation method is proposed in [12]. The approach is based on reformulating the problem as a Petrov-Galerkin (oblique) projection [8], that involves two parameter-dependent transformation matrices. These transformations again rely on the controllability and observability Gramians, but instead of computing them by LMI optimization, [12] proposes an approximation, by using the local Gramians associated with the LTI systems obtained at frozen scheduling parameter values. Since the algorithm uses only QR factorization and singular value decomposition (SVD) it is numerically attractive. On the other hand, it works only for stable systems and the approximation rises some technical questions. Finding answers to these questions is part of the ongoing research.

In the literature some other model reduction methods can also be found for LPV systems, see e.g. [13], [14], [15]. Although these techniques are different, yet, they share a common feature; they are all based on frozen parameter models, i.e. they independently reduce the LTI models obtained at fixed scheduling parameter values and then seek a suitable interpolation algorithm to construct an LPV system from the reduced LTI model set. The latter step is very difficult in general [16], because the independently reduced (transformed and projected) local, LTI systems have to be transformed into a consistent state-space representation.

Regarding parameter-dependent systems, it is important to mention the family of parametric model reduction methods, see e.g. [17], [18], [19], [20]. Though these approaches show similar characteristics to the LPV model reduction, the problem they address is fundamentally different. The parametric model reduction starts from a parameterized set of large-scale LTI systems. The systems are reduced and a model database is constructed from the obtained reduced order models. If a parameter value is given, the corresponding reduced order model is obtained from the stored model set by a suitably chosen interpolation algorithm [21] [22]. It is important to emphasize that the parameters in this framework are considered to be constant in time. This is significantly different from the problem studied in this paper, since here the parameters change in time and thus the system to be reduced is time-varying. Note also that, for LPV systems the input/output equivalence of two models at frozen parameter values, i.e. the input/output equivalence of the corresponding LTI systems, does not imply the input/output equivalence of the two systems along the time varying parameter trajectories [23].

Finally, it has to be mentioned that there is a large family of model reduction tools that have been developed for specific engineering applications. These methods highly exploit the particular properties of the system and the underlying modelling framework, see e.g. the recent papers in [24]. In contrast, the approach presented in this paper belongs to the family of general model reduction methods, as it starts from a general LPV description and no other particular properties are assumed.

The model reduction method proposed in this paper returns to the LPV reduction technique presented in [9], but instead of constructing a numerically tractable approximation for the balanced truncation, it decouples the large-scale system into smaller dimensional LPV subsystems that can be independently reduced by the original algorithms of [9]. The decomposition starts with the construction of a parameter varying modal transformation that separates the system modes. Hierarchical clustering is applied then to group the modes into larger LPV subsystems, which can then be independently reduced. Since the model reduction is applied on LPV subsystems and not on frozen LTI models, the difficulties of system interpolation are avoided. Furthermore, the reduction of unstable systems is naturally integrated in the proposed methodology.

Although the (approximate) modal decomposition is part of the proposed algorithm, it is important to emphasize that the concept is fundamentally different from that of the modal truncation methods elaborated for LTI systems [8], [25]. Modal truncation aims at reducing the large-scale model by keeping only the dominant modal subsystems. This is not efficient in general, because the pole location does not necessarily indicate the contribution of the corresponding state to the input-output behavior of the overall system [8]. This is a reason why we propose to cluster the modal subsystems into larger groups and use a model reduction algorithm to reduce the larger subsystems obtained. The preliminary version of the proposed method have been published in [26] 
and successfully applied in [27] and [28] to generate control oriented model and a model based control for a large-scale flexible wing. In this paper the preliminary concept is refined, the algorithm is improved and several technical details are elaborated.

The paper is organized as follows. In the following section the model reduction problem is formulated. Section 3 is devoted to the construction of the parameter-varying modal transformation. In Section 4 the hierarchical clustering and the balanced reduction methods are discussed. The numerical case studies are presented in Section 5. At the end of the paper the main results are summarized and the most important conclusions are drawn.

\section{PROBLEM FORMULATION}

A continuous-time LPV system can be given in state-space form as follows

$$
G(\rho): \begin{aligned}
& \dot{x}(t)=A(\rho(t)) x(t)+B(\rho(t)) u(t) \\
& y(t)=C(\rho(t)) x(t)+D(\rho(t)) u(t)
\end{aligned}
$$

where $\rho: \mathbb{R}_{+} \rightarrow \mathbb{R}$ denotes the time varying scheduling parameter, $x: \mathbb{R}_{+} \rightarrow \mathbb{R}^{n_{x}}, u: \mathbb{R}_{+} \rightarrow \mathbb{R}^{n_{u}}$ and $y: \mathbb{R}_{+} \rightarrow \mathbb{R}^{n_{y}}$, are respectively the state, input and output. The matrix functions $A: \mathbb{R} \rightarrow$ $\mathbb{R}^{n_{x} \times n_{x}}, B: \mathbb{R} \rightarrow \mathbb{R}^{n_{x} \times n_{u}}, C: \mathbb{R} \rightarrow \mathbb{R}^{n_{y} \times n_{x}}, D: \mathbb{R} \rightarrow \mathbb{R}^{n_{y} \times n_{u}}$ are continuous functions of $\rho$. Furthermore we assume that both $\rho, \dot{\rho}$ and $\ddot{\rho}$ are bounded: $\rho(t) \in \Theta:=\left[\rho_{\min }, \rho_{\max }\right]$ and $\dot{\rho}(t) \in$ $\Omega:=[-\delta, \delta]$ and $\ddot{\rho}(t) \in \Phi:=[-\phi, \phi]$ for all $t^{\dagger}$. (By this definition we restrict ourselves to LPV models having only 1 scheduling parameter. The case of vector valued $\rho$ is commented briefly in the conclusion.)

In order to perform the numerical computations, the LPV system (1) is evaluated over a parameter grid $\Gamma=\left\{\rho_{1}=\rho_{\min }, \rho_{2}, \ldots, \rho_{N}=\rho_{\max }\right\}, \quad \rho_{1}<\rho_{2}<\ldots<\rho_{N}$ and the set $\mathcal{G}$ of LTI systems, obtained at the grid points, is considered:

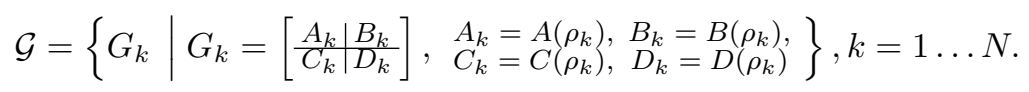

The parameter grid is assumed to be suitably dense such that $\mathcal{G}$ preserves all the significant dynamical properties of the LPV system. More precisely, the dynamical behavior of (1) between any two consecutive grid points can be reconstructed from the LTI models defined at the grid points by using simple (linear, polynomial, piece-wise polynomial (spline)) interpolation algorithm. This grid-based representation is often directly generated by trimming a nonlinear system at different operating points [29].

The aim of the model reduction is to find $G_{r e d}(\rho, \dot{\rho},\{\ddot{\rho}\})$ of order $n_{x}^{r e d} \ll n_{x}$ to be used for designing a model based controller for (1). Therefore, the input-output behavior of the full order model has to be preserved as much as possible. The similarity between the full and the reduced order models can be analyzed by time-domain simulations and by different metrics presented in Section 5. These metrics give quantitative information on the applicability of the reduced order model in model-based control synthesis.

\section{APPROXIMATE MODAL DECOMPOSITION FOR PARAMETER-VARYING SYSTEMS}

\subsection{Modal decomposition in LTI case}

For LTI systems the concept of modal decomposition is theoretically sound. Let the system be given by its state-space matrices $A, B, C, D$ and let $A$ be diagonalizable. Denote $\lambda_{1}, \ldots, \lambda_{m}$ the eigenvalues of $A$ such that the complex-conjugate pairs are grouped together, i.e. for a complex eigenvalue $\lambda_{i}=r_{i} \pm c_{i} j$. Then there exists a similarity state transformation $\bar{T}$ such that the ${ }^{\dagger}$ For parameter-dependent variables we use the following notational convention: $a(\rho)$ denotes the variable $a$ as a function
of the parameter $\rho$, while $a(\rho(t))$ or $a\left(\rho_{k}\right)$ denotes the value of the variable at the specific parameter value $\rho(t)$ or $\rho_{k}$. 
matrix $\bar{A}=\bar{T}^{-1} A \bar{T}$ is block diagonal in the form $\bar{A}=\operatorname{blockdiag}\left(\bar{A}_{1}, \ldots, \bar{A}_{m}\right)$, where each $\bar{A}_{i}$ corresponds to a real eigenvalue or a complex-conjugate eigenvalue pair and $\bar{A}_{i}=\lambda_{j}$ if $\lambda_{j}$ is real and $\bar{A}_{i}=\left[\begin{array}{cc}r_{i} & c_{i} \\ -c_{i} & r_{i}\end{array}\right]$ if $\lambda_{i}$ is complex. The similarity transformation $\bar{T}$ is constructed from the eigenvectors as follows. Let $v_{1}, \ldots v_{m}$ be the eigenverctors (real and complex conjugate eigenvector pairs) corresponding to eigenvalues above. Then $\bar{T}=\left[\nu_{1}, \ldots, \nu_{m}\right]$, where $\nu_{i}=v_{i}$ if $\lambda_{i}$ is real and $\nu_{i}=\left[\operatorname{Re}\left(v_{i}\right) \operatorname{Im}\left(v_{i}\right)\right]$ if $\lambda_{i}$ is complex. With the block diagonal $\bar{A}$ the original system decouples into the modal subsystems $\left(\bar{A}_{i}, \bar{B}_{i}, \bar{C}_{i}, D\right)$, where $\bar{B}_{i}$ and $\bar{C}_{i}$ denote the sub-blocks of $\bar{T}^{-1} B$ and $C \bar{T}$ corresponding to $\bar{A}_{i}$. Note that $\bar{T}$ is not unique, because it can be constructed from any eigenvectorset spanning the same eigen-space.

Our aim is to extend this concept to parameter-varying systems and construct a parameterdependent state transformation $\bar{T}(\rho)$ that decouples (at least approximately) the LPV system into parameter-varying modal subsystems.

\subsection{Simplifying assumptions}

In order to simplify the discussion we consider first a special case, when matrix $A(\rho)$ satisfies a set of technical conditions. Later, it is shown that most of these conditions can be relaxed. Now let the following assumptions be made:

(A) $A(\rho)$ is diagonalizable and has a differentiable eigenvalue decomposition, i.e. there exist a diagonal $\Lambda(\rho)$ and invertible $V(\rho)$ matrices such that both are differentiable in $\rho$ and $A(\rho)=V(\rho) \Lambda(\rho) V(\rho)^{-1}$. The diagonal entries $\lambda_{i}(\rho)$ of $\Lambda(\rho)\left(i=1 \ldots n_{x}\right)$ are the parameterdependent eigenvalues, while the columns $v_{i}(\rho)$ of $V(\rho)\left(i=1 \ldots n_{x}\right)$ give the parameterdependent eigenvectors.

(B) The characteristics of each $\lambda_{i}(\rho)$, i.e. its multiplicity and type (complex or real) does not change over the parameter domain.

Assumption (A) implies first that each $A_{k}$ matrix is diagonalizable, so the eigen-decomposition $\Lambda_{k}=V_{k}^{-1} A_{k} V_{k}$ exists in every grid point. Second, the analyticity of $V(\rho)$ makes it possible to construct a differentiable and invertible parameter-varying state transformation from the eigenvectors $V_{k}$. Assumption (B) guarantees that the dimension of the eigenspace associated with each eigenvalue is constant for all scheduling parameter value. This makes it easier to transform the eigenvectors $V_{k}$ into a smooth sequence, from which a differentiable state transformation can be constructed.

\subsection{Eigen-decomposition}

Our aim is to construct the differentiable $\lambda_{i}(\rho), v_{i}(\rho)$ functions satisfying $\lambda_{i}\left(\rho_{k}\right)=\lambda_{k, i}$ and $v_{i}\left(\rho_{k}\right)=v_{k, i}$, where $\Lambda_{k}=\operatorname{blockdiag}\left(\lambda_{k, 1}, \ldots, \lambda_{k, n_{x}}\right)$ and $V_{k}=\left[v_{k, 1}, \ldots, v_{k, n_{x}}\right]$ define the eigendecomposition of $A_{k}$ such that

$$
\Lambda_{k}=V_{k}^{-1} A_{k} V_{k}, \quad k=1 \ldots N
$$

Note that, if the eigen-decomposition are computed for each $A_{k}$ the ordering of the eigenvalues obtained may vary over the grid points, so the sequence $\lambda_{1, i}, \ldots, \lambda_{N, i}$ may not correspond to $\lambda_{i}\left(\rho_{1}\right), \ldots, \lambda_{i}\left(\rho_{N}\right)$. In order to ensure the consistency of the $\Lambda_{k}$ matrices, the ordering of the eigenvalues has to be modified in each grid point. The right ordering can be found if the eigenvalues at every grid point are correctly paired with the eigenvalues at the succeeding grid point. To find this pairing, two ingredients are needed: first, a distance metric to compare the eigenvalues and second, an algorithm to find the pairing. Moreover, the algorithm should work efficiently for large number of eigenvalues as well. In the next section a suitably distance metric is proposed, which compares the eigenvalues based on dynamic similarity. Then the pairing problem is reformulated as finding a perfect matching in a bipartite graph. This is beneficial, because the latter problem can be solved efficiently in polynomial time. 
Hyperbolic distance metric. Our goal is to connect the series of local eigenvalues in such way that the resulting continuous trajectories correspond to the parameter-varying eigenvalues of the $A(\rho)$ matrix. In case of an LTI system each eigenvalue represents a dynamical mode, i.e. a subsystem with particular dynamical characteristics. Extending this concept to the parameter varying case, we intend to pair two eigenvalues if they belong to the same parameter varying subsystem. For this purpose the hyperbolic distance metric is adopted, which measures dynamic similarity between the eigenvalues. The reasons why this metric is chosen are detailed in the appendix. On the other hand, this metric compares only the eigenvalues. In order to take the associated eigenvectors into consideration as well, the distance metric is weighted by the modal assurance criterion (MAC) [30] measuring the directional similarity of the normalized eigenvectors. Formally, if the weighted distance metric is denoted by $h_{w}(\cdot, \cdot)$ the final formula we use to compare two eigenvalues, e.g. $\lambda_{k, i}$ and $\lambda_{k+1, j}$ (and their eigenvectors $v_{k, i}$ and $v_{k+1, j}$ ) can be given as follows:

$$
h_{w}\left(\lambda_{k, i}, \lambda_{k+1, j}\right):=h\left(\lambda_{k, i}, \lambda_{k+1, j}\right) \cdot\left(1-\left|v_{k, i}^{*} v_{k+1, j}\right|\right)
$$

where $h(\cdot, \cdot)$ is defined in the appendix.

Perfect matching in complete bipartite graphs. Let $\mathcal{L}_{k}$ and $\mathcal{L}_{k+1}$ denote the ordered sets containing the eigenvalues at grid points $k$ and $k+1$, respectively, i.e. $\mathcal{L}_{k}=\left\{\lambda_{k, 1}, \ldots, \lambda_{k, n_{x}}\right\}, \mathcal{L}_{k+1}=$ $\left\{\lambda_{k+1,1}, \ldots, \lambda_{k+1, n_{x}}\right\}$. In order to pair every eigenvalue in $\mathcal{L}_{k}$ with exactly one eigenvalue in $\mathcal{L}_{k+1}$ and vice versa, a graph-theoretic reformulation is applied. For this, let the weighted, complete, bipartite graph [31] $\mathcal{B}_{k}=\left(\mathcal{N}_{k}, \mathcal{E}_{k}, \mathcal{C}_{k}\right)$ be defined with vertices $\mathcal{N}_{k}=\mathcal{L}_{k} \cup \mathcal{L}_{k+1}$, edges $\mathcal{E}_{k}=$ $\left\{e_{i j} \mid e_{i j}:=\left(\lambda_{k, i}, \lambda_{k+1, j}\right), \forall(i, j)\right.$ pairs $\}$ and edge-costs $\mathcal{C}_{k}=\left\{c_{i j} \mid c_{i j}=h_{w}\left(\lambda_{k, i}, \lambda_{k+1, j}\right)\right\}$. So $\mathcal{B}_{k}$ is defined such that its edges connect every element in $\mathcal{L}_{k}$ with all elements in $\mathcal{L}_{k+1}$ and the cost of an edge characterizes the dynamical similarity between the two eigenvalues on the edge. Note that every $\lambda_{k, i}$ has to have exactly one pair in $\mathcal{L}_{k+1}$ and every $\lambda_{k+1, j}$ has to be a pair of exactly one vertex in $\mathcal{L}_{k}$. In terms of the graph-theoretic setup this is a perfect matching ([32], [31]) in $\mathcal{B}_{k}$, characterized by a set of $n_{x}$ independent edges in $\mathcal{E}_{k}$. The cost of a perfect matching is defined by the sum of the costs for the corresponding set of independent edges. Accordingly, finding the right pairing between the eigenvalues of $\mathcal{L}_{k}$ and $\mathcal{L}_{k+1}$ is formulated as finding the minimum cost perfect matching in $\mathcal{B}_{k}$ [32]. The obtained matching problem can be efficiently solved in polynomial $\left(\mathcal{O}\left(n_{x}^{3}\right)\right)$ time by using the Hungarian Method (Kuhn-Munkres Algorithm) [31], offering a numerically attractive solution for the eigenvalue pairing problem. Considering the ordering of the eigenvalues at $\rho_{1}$ as a reference, the pairing problem can be solved successively for $k=1,2, \ldots, N-1$. As a result, the $\mathcal{L}_{k}$ sets (and consequently the $\Lambda_{k}$ matrices) will be consistently ordered. This ordering has to be applied to the columns of $V_{k}$ as well, in order that the eigenvectors become consistent with the reordered eigenvalues.

\subsection{Continuity of the eigenvectors and the Procrustes-problem}

The next step is to shape the eigenvectors stored in $V_{k}$, such that their entries form a smooth function along the parameter grid. This is necessary to interpolate the entries of $V_{k}$ into a smooth, differentiable function and thus facilitating the construction of a differentiable and modal transformation. Of course, the shaping of $V_{k}$ has to preserve the eigenspace spanned by the eigenvectors.

To this end, the first step is to identify the multiplicity of the eigenvalues in order that the eigenvectors associated with a same eigenvalue can be grouped and handled together. For this, let $\tau_{i}$ denote the $i$-th eigenvalue trajectory, i.e. $\tau_{i}=\left(\lambda_{1, i}, \ldots, \lambda_{N, i}\right)$. By using (22), we can introduce a distance metric between two eigenvalue trajectories as follows ${ }^{\ddagger}$ :

$$
H\left(\tau_{i}, \tau_{j}\right)=\min \left(\max _{k} h\left(\lambda_{k, i}, \lambda_{k, j}\right), \max _{k} h\left(\lambda_{k, i}, \lambda_{k, j}^{*}\right)\right) .
$$

\footnotetext{
${ }^{\ddagger}$ Note that, (5) can also be defined by the weighted $h_{w}(\cdot, \cdot)$ metric as well.
} 
This metric does not distinguish the complex pairs, i.e. the distance of $\tau_{i}$ from $\tau_{j}$ and $\tau_{j}^{*}$ are the same. This is important to render the complex conjugate eigenvalue sequences together. By computing the distance between every $\left(\tau_{i}, \tau_{j}\right)$ pair, the multiple eigenvalue trajectories can be identified. Two eigenvalue trajectories are considered to belong to a single repeated eigenvalue, if the distance between them is smaller than a given threshold. $\$$

By assigning to every eigenvalue the eigenvectors spanning its eigenspace, an ordered set $\left\{\left(\lambda_{k, 1}, V_{k, 1}\right), \ldots,\left(\lambda_{k, n}, V_{k, n}\right)\right\}, n \leq n_{x}$ can be defined at each grid point. Here $V_{k, i} \in \mathbb{C}^{n_{x} \times d_{i}}$ collects the eigenvectors associated with $\lambda_{k, i}$. Due to assumption (B) the dimension $d_{i}$ of the eigenspace associated with the $i$-the eigenvalue is constant and the same at every grid point, for any $i$. This property will be exploited in the algorithm below.

The next step is to transform the eigenvector sequence $V_{1, i}, \ldots, V_{N, i}$ for all $i$. This can be done by a right multiplication of $V_{k, i}$ with an invertible matrix $Q_{k, i}$, which changes the eigenvectors, however leaves the eigenspace intact. To obtain the required smooth interpolation, $Q_{k, i}$ should transform the respective eigenvectors at consecutive grid points as close as possible. This condition is formulated as a complex, unconstrained Procrustes problem [33], [18], [22] as follows:

$$
\bar{Q}_{k+1, i}:=\arg \min _{Q_{k+1, i}}\left\|V_{k, i}-V_{k+1, i} Q_{k+1, i}\right\|_{F}, \quad \forall k, i
$$

where $k$ goes from 1 to $N-1, i \in\{1, \ldots, n\}, Q_{k+1, i} \in \mathbb{C}^{d_{i} \times d_{i}}$. The solution for (6) can be analytically given in the following closed form:

$$
\operatorname{vec}\left(\bar{Q}_{k+1, i}\right)=\left(I \otimes\left[\begin{array}{cc}
\operatorname{Re}\left(V_{k+1, i}\right) & -\operatorname{Im}\left(V_{k+1, i}\right) \\
\operatorname{Im}\left(V_{k+1, i}\right) & \operatorname{Re}\left(V_{k+1, i}\right)
\end{array}\right]\right)^{\dagger} \operatorname{vec}\left(\left[\begin{array}{c}
\operatorname{Re}\left(V_{k, i}\right) \\
\operatorname{Im}\left(V_{k, i}\right)
\end{array}\right]\right)
$$

where $\operatorname{vec}\left(\bar{Q}_{k+1, i}\right)$ is a vector formed from $\bar{Q}_{k+1, i}$ by stacking its columns below each other. Accordingly, $\bar{V}_{k+1, i}=V_{k+1, i} \bar{Q}_{k+1, i}$ is the appropriately rotated eigenvector, consistent with $V_{k, i}$. The described Procrustes problem hence can be solved successively for each eigenvalue of the system over the parameter domain $k=1,2, \ldots N$. It should be noted, that the Procrustes iteration can be started from any other grid point as well. The iteration has to be performed then in both directions: for the larger and for the smaller indices. 9

\subsection{Approximate modal transformation}

Due to the corrections above, the sequence $\bar{V}_{1}, \ldots \bar{V}_{N}$ of the shaped eigenvector matrices (with $\bar{V}_{k}=$ $\left[\begin{array}{lll}\bar{V}_{k, 1} \ldots \bar{V}_{k, n}\end{array}\right]$ ) can be smoothly interpolated (e.g. by linear, polynomial or piecewise polynomial (spline) interpolation) over the parameter domain. From the resulted $\bar{V}(\rho)$ matrix valued function the parameter dependent, differentiable transformation $\bar{T}(\rho)$ can be obtained in the same way as in the LTI case. Defining a new state vector $\bar{x}$ such that $\bar{T}(\rho) \bar{x}=x$, the original LPV system (1) transforms into

$$
\begin{aligned}
\dot{\bar{x}} & =\left(\bar{T}^{-1}(\rho) A(\rho) \bar{T}(\rho)-\bar{T}^{-1}(\rho) \frac{\partial \bar{T}(\rho)}{\partial \rho} \dot{\rho}\right) \bar{x}+\bar{T}^{-1}(\rho) B(\rho) u \\
& =(\bar{A}(\rho)+\bar{E}(\rho, \dot{\rho})) \bar{x}+\bar{B}(\rho) u \\
y & =C(\rho) \bar{T}(\rho) \bar{x}+D(\rho) u=\bar{C}(\rho) \bar{x}+D(\rho) u .
\end{aligned}
$$

where $\bar{A}(\rho)$ is the block diagonal part of $\bar{T}^{-1}(\rho) A(\rho) \bar{T}(\rho)$ such that $\bar{A}\left(\rho_{k}\right)=\bar{T}^{-1}\left(\rho_{k}\right) A\left(\rho_{k}\right) \bar{T}\left(\rho_{k}\right)$ for all $\rho_{k} \in \Gamma . \bar{E}(\rho, \dot{\rho})$ collects the $\dot{\rho}$-dependent terms in (8) and the difference $\bar{T}^{-1}(\rho) A(\rho) \bar{T}(\rho)-$

\footnotetext{
$\S$ This threshold-based decision is necessary here, because after the numerical manipulations (e.g. the eigendecomposition) performed on the LPV model we might not expect that the eigenvalue sequences corresponding to a multiple eigenvalue of $A(\rho)$ will be perfectly equal in each grid point and give 0 distance. On the other hand, if the original system contains distinct eigenvalues that are very close to each other, then from numerical point of view, it is generally better to handle them as a single, repeated eigenvalue. The threshold is typically chosen close to the numerical precision of the computer arithmetic; for example in the case studies we applied $100 \epsilon$, where $\epsilon$ is the relative accuracy of the floating point numerical operations in MATLAB, that is $\epsilon=2.22 \cdot 10^{-16}$.

ฯ It is a reasonable to analyze the numerical conditioning of the eigenvector matrices and choose the starting point, where the associated $V_{k}$ matrix is well-conditioned.
} 
$\bar{A}(\rho)$ for all $\rho \in \Theta$. The latter is zero only at the grid points. The term $\bar{E}(\rho, \dot{\rho})$ represents the main challenge in the application of the transformation, since it represents cross-coupling between the modal subsystems. Without it, the transformed system would be fully decoupled and similar to the LTI modal form. Therefore, at this point the transformed model has to be analyzed in order to decide if $\bar{E}(\rho, \dot{\rho})$ has to be kept or can be neglected. If the original and the modal system do not differ significantly in terms of input-output behavior, the $\dot{\rho}$-dependent term can be dropped out and the computations can be proceeded with the decoupled structure. Otherwise, it has to be kept and taken into account in the final, model reduction step as discussed in Section 4.2.

Note that, the presented modal decomposition allows the identification of modes (parameter varying subsystems) with distinguished characteristics, such as integrators, unstable and mixed stability modes, etc. These modes are often represent key system properties so it is necessary to preserve them in the reduced order model. Using the modal form these modes can be temporarily removed from the full order model and after reduction they can be added back to the reduced system. Similarly, fast modes outside of the operating domain of interest can also be eliminated at this step.

\subsection{Relaxation of conditions $A$ and $B$}

Clearly, if $A(\rho)$ has a differentiable eigen-decomposition and one can give a numerical algorithm that constructs it, there is no need for the most steps above and the modal transformation can easily determined from the parameter-dependent eigenvectors. The eigen-decomposition of parametervarying matrices has been subject of intensive research for decades, see e.g. [34], [35], [36], [37], [38], [39]. These papers provide rigorous mathematical conditions for the existence of an analytic eigenvalue/eigenvector system and point out the difficulties of their numerical computation.

Before identifying these difficulties as critical weak points of our approach, two important comments have to be made. First, the construction of the modal transformation requires only that $\bar{V}(\rho)$ be differentiable and suitably smooth function such that $\bar{V}^{-1}\left(\rho_{k}\right) \operatorname{diag}\left(\lambda_{k, 1} \ldots \lambda_{k, n_{x}}\right) \bar{V}\left(\rho_{k}\right)=$ $A\left(\rho_{k}\right)$ holds at every grid point $\rho_{k} \in \Gamma$. It is therefore not necessary that $\bar{V}(\rho)$ reconstructs exactly the true eigenvector functions of $A(\rho)$. Second, it also has to be kept in mind that the LPV model (1) is always only an approximation of the underlying physical system, hence it is possible to apply numerical perturbations in the systems description, as long as the overall input/output behavior does not change significantly. Therefore, small perturbations on the $A(\rho)$ matrix is allowed as long as they do not significantly influence the input/output map realized by the dynamical system.

If condition B does not hold a small perturbation can help again, but in this case a more systematic way is also available. The eigenvalue trajectories that cross at certain parameter values can be grouped and handled together. The associated eigenvectors are then smoothed simultaneously by a full block matrix in the Procrustes algorithm. Consequently, a larger dimensional subsystem (comprising the grouped eigenvalues) appears in the modal form, which is then handled as a single, indivisible object in the next, clustering step.

The case study in Section 5.1 gives an example for the application of the ideas above in practice.

\section{CLUSTERING AND BALANCED REDUCTION}

The algorithm we have developed so far is able to decouple (at least approximately) the LPV system into a set of independent parameter-varying modal subsystems. Next, the idea is to group these modal blocks with similar dynamical properties into clusters, so that the corresponding larger dimensional subsystems can be efficiently reduced. The clustering is based on the eigenvalue trajectories $\tau_{1} \ldots \tau_{n_{x}}$ constructed in section 3.4 , so the coupling term $\bar{E}(\rho, \dot{\rho})$, either significant or not, is neglected in this step. If the effect of $\bar{E}(\rho, \dot{\rho})$ is not negligible, it can be taken into consideration later at the final model reduction phase, in section 4.2.

In the forthcoming two subsections, we show first how the hierarchical agglomerative clustering (HAC) methodology [40], [41] can be adapted to solve our specific clustering problem. Then, based on [9], the main steps of the parameter-varying balanced reduction algorithm are recalled. 


\subsection{Hierarchical clustering}

In this section the hierarchical agglomerative clustering methodology is adopted to group the similar modal subsystems together. The HAC framework is a bottom-up clustering approach, where each data object is treated as a singleton cluster and successively merged until a single cluster is obtained.

In the context of the model reduction, the eigenvalue trajectories $\tau_{i}, i=1, \ldots, n_{x}$ are considered as the individual data objects. For measuring similarities between these trajectories, formula (5) is used. As this metric ensures merging of complex pairs into one cluster, the parameter-varying modes $-\left(\tau_{i}, \tau_{i}^{*}\right)$ pairs - take place at the lowest level of the HAC. For the comparison of two clusters the complete link clustering is applied, i.e.: the similarity of two clusters is determined by the similarity of their most dissimilar members. Formally, if $C_{m}$ and $C_{n}$ are two clusters, then the corresponding merging criterion is:

$$
L\left(C_{m}, C_{n}\right)=\left\{\max _{i, j}\left(H\left(\tau_{i}, \tau_{j}\right)\right), \tau_{i} \in C_{m}, \tau_{j} \in C_{n}\right\} .
$$

Consequently, in the HAC framework, at each algorithmic step those two clusters are merged together for which the (9) value is the smallest. Note that (9) is non-local, i.e. the entire structure of the clustering can influence merge decisions. This results in a preference for compact clusters with small diameters (i.e. the most similar dynamics are grouped together) and causes sensitivity to outliers (i.e. uncommon dynamical components). The merging is repeated until all the objects have been grouped into a single cluster. This can be done in $O\left(n_{x}^{2} \log n_{x}\right)$ steps [40]. The result of the HAC is visualized by a dendrogram, which is a tree diagram illustrating how the data objects are merged into larger clusters until the one single cluster is reached. The final cluster structure is obtained by cutting the dendrogram at a user-defined level of similarity. The careful choice of this threshold is important, because it determines the number and size of the clusters generated. In the decomposition of dynamical systems the number of clusters is mainly limited by the size of the largest cluster, since the cluster size gives the dimension of the underlying dynamical system, which cannot be arbitrarily large due to the numerical limitations of the balanced reduction algorithm to be applied in the next step.

Assume $M$ clusters have been generated. By rearranging the state $\bar{x}$ of (8) according to these clusters the obtained system structure is illustrated in Fig. 1. If the new state vector is denoted by $\tilde{x}$ and $\Pi$ is the permutation matrix mapping $\tilde{x}$ to $\bar{x}$, i.e. $\bar{x}=\Pi \tilde{x}$, then

$$
\begin{array}{rlrl}
\tilde{A}(\rho) & =\Pi^{T} \bar{A}(\rho) \Pi, & \tilde{B}(\rho)=\Pi^{T} \bar{B}(\rho) \\
\tilde{E}(\rho, \dot{\rho}) & =\Pi^{T} \bar{E}(\rho, \dot{\rho}) \Pi & & \\
\tilde{C}(\rho) & =\bar{C}(\rho) \Pi, & \tilde{D}(\rho)=D(\rho)
\end{array}
$$

Without $\tilde{E}(\rho, \dot{\rho})$ the dynamics are fully decoupled into $M$ subsystems $\tilde{G}^{(\ell)}(\rho)=$ $\left[\begin{array}{c|c}\tilde{A}^{(\ell)}(\rho) & \tilde{B}^{(\ell)}(\rho) \\ \hline \tilde{C}^{(\ell)}(\rho) & D^{(\ell)}(\rho)\end{array}\right], \ell=1, \ldots, M$. The dimension of each subsystem equals to the size of the corresponding cluster. If we have decided earlier to neglect the coupling term $\tilde{E}(\rho, \dot{\rho})$, then these subsystems can be handled separately. Otherwise, the coupling term has to be taken into consideration. Since a decoupled structure is needed to continue our algorithm, this is only partially possible. For this, let $\tilde{E}(\rho, \dot{\rho})$ be expressed as a sum of $\tilde{E}_{1}(\rho, \dot{\rho})$ and $\tilde{E}_{2}(\rho, \dot{\rho})$ such that the structure of $\tilde{E}_{1}(\rho, \dot{\rho})$ is aligned with the structure of $\tilde{A}$ (see Fig. 1). Assuming that the effect of $\tilde{E}_{2}(\rho, \dot{\rho})$ on the input-output behavior is negligible, we can proceed the computations with the subsystems defined by the system matrices $\tilde{A}^{(\ell)}(\rho)+\tilde{E}_{1}^{(\ell)}(\rho, \dot{\rho}), \tilde{B}^{(\ell)}(\rho), \tilde{C}^{(\ell)}(\rho)$ and $D^{(\ell)}(\rho)$. These systems are also independent, so they can be separately reduced. The neglected term can be treated as a modeling uncertainty during the analysis or control synthesis procedures performed on the reduced order model. \| "To further minimize the approximation error, it is a reasonable idea to complete the distance metric (5) with an additional
term penalizing large entries in $\tilde{E}_{2}(\rho, \dot{\rho})$. 


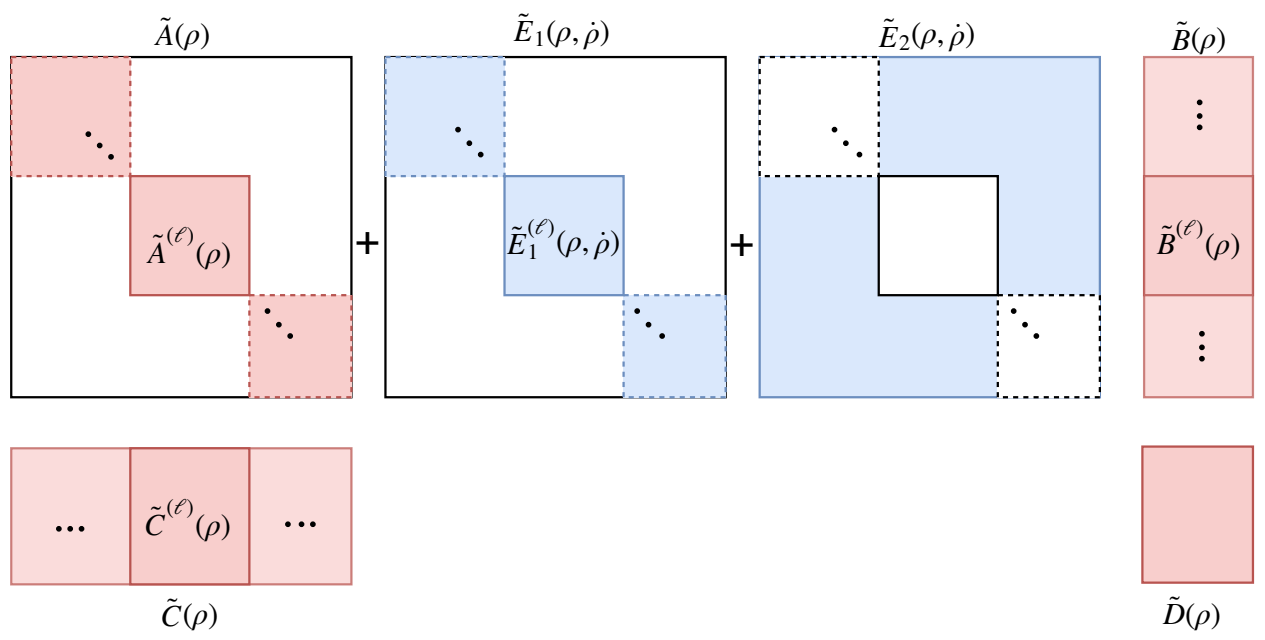

Figure 1. The structure of the system matrices after clustering: $\tilde{A}(\rho)=\operatorname{blockdiag}\left(\tilde{A}^{(1)}(\rho), \ldots, \tilde{A}^{(M)}(\rho)\right)$, $\tilde{E}(\rho, \dot{\rho})$ is decomposed such that $\tilde{E}(\rho, \dot{\rho})=\tilde{E}_{1}(\rho, \dot{\rho})+\tilde{E}_{2}(\rho, \dot{\rho})$ such that the structure of $\tilde{E}_{1}(\rho, \dot{\rho})$ is aligned with the structure of $\tilde{A}(\rho)$. The white areas denote the zero entries.

\subsection{Balanced reduction}

Balanced reduction is a fundamental approach for the model reduction of linear (time invariant and varying, as well as parameter-dependent) systems [10], [9]. The key concept is the balanced realization which reveals the controllability and observability properties of the system. After clustering the dynamical modes, $M$ separate LPV systems are obtained, each of which can be given in the following general form:

$$
\begin{aligned}
& \dot{x}^{(\ell)}=\tilde{A}^{(\ell)}(\tilde{\rho}) \tilde{x}^{(\ell)}+B^{(\ell)}(\tilde{\rho}) u \\
& y^{(\ell)}=\tilde{C}^{(\ell)}(\tilde{\rho}) x^{(\ell)}+D^{(\ell)}(\tilde{\rho}) u,
\end{aligned}
$$

where $\tilde{A}^{(\ell)}(\tilde{\rho})=\tilde{A}^{(\ell)}(\rho)+\tilde{E}_{1}(\rho, \dot{\rho})$ and $\tilde{\rho}=[\rho, \dot{\rho}]$. The similarity transformation $\hat{T}(\tilde{\rho})$, which transforms (11) into balanced form is obtained from the observability $X_{o}^{(\ell)}(\tilde{\rho})$ and controllability $X_{c}^{(\ell)}(\tilde{\rho})$ Gramians**. If the LPV system is given in a state-space form and the structure of the Gramians is a-priori fixed (e.g. in the form $X_{o}^{(\ell)}(\tilde{\rho})=X_{o, 0}^{(\ell)}+\sum_{i=1}^{n_{b}} X_{o, i}^{(\ell)} f_{i}(\tilde{\rho}) X_{o, i}^{(\ell)}$ and $X_{c}^{(\ell)}(\tilde{\rho})=$ $X_{c, 0}^{(\ell)}+\sum_{i=1}^{n_{b}} X_{c, i}^{(\ell)} g_{i}(\tilde{\rho}) X_{c, i}^{(\ell)}$ where $f_{i}(\tilde{\rho}), g_{i}(\tilde{\rho})$ are fixed basis functions and $X_{o, i}^{(\ell)}, X_{c, i}^{(\ell)}, i=$ $1, \ldots n_{b}$ are free variables), then $X_{o}^{(\ell)}(\tilde{\rho})$ and $X_{c}^{(\ell)}(\tilde{\rho})$ can be obtained as a result of the following optimization problem [9]:

$$
\begin{array}{r}
\min _{X_{o, i}^{(\ell)}, X_{c, i}^{(\ell)}, i=1 \ldots n_{b}} \sum_{k} \operatorname{trace} X_{o}^{(\ell)}\left(\tilde{\rho}_{k}\right) X_{c}^{(\ell)}\left(\tilde{\rho}_{k}\right) \\
\dot{X}_{o}^{(\ell)}\left(\tilde{\rho}_{k}, \tilde{\nu}_{s}\right)+A^{(\ell)}\left(\tilde{\rho}_{k}\right)^{T} X_{o}^{(\ell)}\left(\tilde{\rho}_{k}\right)+X_{o}^{(\ell)}\left(\tilde{\rho}_{k}\right) A^{(\ell)}\left(\tilde{\rho}_{k}\right)+C^{(\ell)}\left(\tilde{\rho}_{k}\right)^{T} C^{(\ell)}\left(\tilde{\rho}_{k}\right) \prec 0 \\
-\dot{X}_{c}^{(\ell)}\left(\tilde{\rho}_{k}, \tilde{\nu}_{s}\right)+A^{(\ell)}\left(\tilde{\rho}_{k}\right) X_{c}^{(\ell)}\left(\tilde{\rho}_{k}\right)+X_{c}^{(\ell)}\left(\tilde{\rho}_{k}\right) A^{(\ell)}\left(\tilde{\rho}_{k}\right)^{T}+B^{(\ell)}\left(\tilde{\rho}_{k}\right) B^{(\ell)}\left(\tilde{\rho}_{k}\right)^{T} \prec 0 \\
X_{o}^{(\ell)}\left(\tilde{\rho}_{k}\right) \succ 0, X_{c}^{(\ell)}\left(\tilde{\rho}_{k}\right) \succ 0, \forall \tilde{\rho}_{k} \in \tilde{\Theta} \text { and } \forall \tilde{\nu}_{s} \in \tilde{\Omega}
\end{array}
$$

where $\tilde{\Theta}$ and $\tilde{\Omega}$ are suitably dense grids over $\Theta \times \Omega$ and $\Omega \times \Phi$, respectively. This is a nonconvex optimization problem, but if either $X_{o}^{(\ell)}\left(\tilde{\rho}_{k}\right)$ or $X_{c}^{(\ell)}\left(\tilde{\rho}_{k}\right)$ is fixed, then the cost function becomes

** The presented balanced reduction algorithm can only be applied to quadratically stable LPV systems. The extension of the method to unstable systems is well documented in [9] and [10]. If the unstable and mixed stability modes are previously identified and separated in the modal form (see Section 3.5), then only the stable part of the system has to be reduced, so the algorithm above can be applied. 
linear in the remaining variables, hence the problem reduces to a linear optimization problem with (LMI) constraints. As suggested in the literature by alternately fixing $X_{o}^{(\ell)}\left(\tilde{\rho}_{k}\right)$ and $X_{c}^{(\ell)}\left(\tilde{\rho}_{k}\right)$ a numerically tractable iterative algorithm is obtained, where an initial $X_{o}^{(\ell)}\left(\tilde{\rho}_{k}\right)$ (or $X_{c}^{(\ell)}\left(\tilde{\rho}_{k}\right)$ ) can be calculated from the frozen parameter solutions.

Although the above modifications ease the computational burden, the approach still suffers from the curse of dimensionality. The number of decision variables involved, and hence the numerical complexity grows with the state-dimension of the system. If the number of states goes over 30-40, the LMI optimization problem becomes intractable by the currently available semidefinite solvers. The decomposition of the system into smaller, independent subsystems, offers a remedy for this problem.

Having determined the observability and controllability gramians of every subsystem, the balancing $\hat{T}^{(\ell)}(\tilde{\rho})$ transformations and the corresponding parameter dependent, generalized singular value trajectories can be determined. The details of the related numerical algorithms can be found in [9]. Applying the balancing transformations on the subsystems the states corresponding to small singular values can be then eliminated. Since the $\hat{T}^{(\ell)}(\tilde{\rho})$ transformations depend on $\rho$ and $\dot{\rho}$, the reduced systems explicitly depend on $\dot{\rho}$ and $\ddot{\rho}$ as well [9]. ${ }^{\dagger \dagger}$

Having reduced the subsystems individually, the reduced dynamics are finally joined together to obtain the low dimensional approximation of (2). To formally express the result, let the balanced state vector of the $\ell$-th subsystem be denoted by $\hat{x}^{(\ell)}$ and let $\hat{T}^{(\ell)}(\tilde{\rho})$ be defined such that $\tilde{x}^{(\ell)}=\hat{T}^{(\ell)}(\tilde{\rho}) \hat{x}^{(\ell)}$. Then, by definition the state $z$ of the reduced order model can be computed as $z=\hat{\Pi}^{T} T^{-1}(\tilde{\rho}) \tilde{x}$, where $\hat{T}(\tilde{\rho})=\operatorname{blockdiag}\left(\hat{T}^{(1)}(\tilde{\rho}), \ldots, \hat{T}^{(M)}(\tilde{\rho})\right)$,

$$
\begin{aligned}
\hat{\Pi} & =\operatorname{blockdiag}\left(\hat{\Pi}^{(1)}, \ldots, \hat{\Pi}^{(M)}\right) \\
\hat{\Pi}^{(\ell)} & =\left[I_{\operatorname{dim}\left(z^{(\ell)}\right)} 0\right]^{T}, \text { such that } z^{(\ell)}=\left(\hat{\Pi}^{(\ell)}\right)^{T} \hat{x}^{(\ell)},
\end{aligned}
$$

and $z^{(\ell)}$ denotes the state of the $\ell$-th reduced subsystem. Define $W(\tilde{\rho})=\hat{\Pi}^{T} \hat{T}^{-1}(\tilde{\rho})$ and $V(\tilde{\rho})=$ $\hat{T}(\tilde{\rho}) \hat{\Pi}$. Then the reduced order model can be expressed as follows:

$$
\begin{aligned}
\dot{z} & =\left(W(\tilde{\rho}) \tilde{A}(\tilde{\rho}) V(\tilde{\rho})-W(\tilde{\rho}) \dot{V}(\tilde{\rho})+W(\tilde{\rho}) \tilde{E}_{2}(\tilde{\rho}) V(\tilde{\rho})\right) z+W(\tilde{\rho}) \tilde{B} u \\
y & =\tilde{C}(\tilde{\rho}) V(\tilde{\rho}) z+\tilde{D}(\tilde{\rho}) u
\end{aligned}
$$

Since term $\tilde{E}_{2}$ has been neglected in the reduction procedure, the term $W(\tilde{\rho}) \tilde{E}_{2}(\tilde{\rho}) V(\tilde{\rho})$ appears in the reduced order model. This term can be considered as an additive uncertainty that has to be taken into consideration during the model based control synthesis.

\section{CASE STUDIES}

\subsection{A benchmark example}

As a first example a 80 dimensional, 2-input-2-output LPV system has been generated for the numerical evaluation of the developed algorithm. The procedure used to generate the model consists of 4 steps. First, the parameter-variation of each eigenvalue is defined over the parameter domain $\Omega=[0,1]$. The obtained $\lambda_{i}(\rho)$ functions are then used for constructing a parameter-varying blockdiagonal $A_{0}(\rho) \in \mathbb{R}^{80 \times 80}$ matrix. This is then completed with a randomly generated constant input $B_{0} \in \mathbb{R}^{80 \times 2}$ and output $C_{0} \in \mathbb{R}^{2 \times 80}$ mappings, as well as a constant direct feedthrough term $D_{0} \in \mathbb{R}^{2 \times 2}$. To make the problem more realistic and industrially relevant, the generated modal system is transformed in the second step by a parameter-varying matrix $T(\rho) . T(\rho)$ is constructed by randomly generating an invertible matrix and making its certain (randomly selected) blocks $\dagger \dagger$ If the second derivative of $\rho$ is not bounded or its bounds are unknown, then by simply removing the $\dot{\rho}$-dependence of
the Gramians also eliminates the explicit $\ddot{\rho}$-dependence of the reduced order system. 


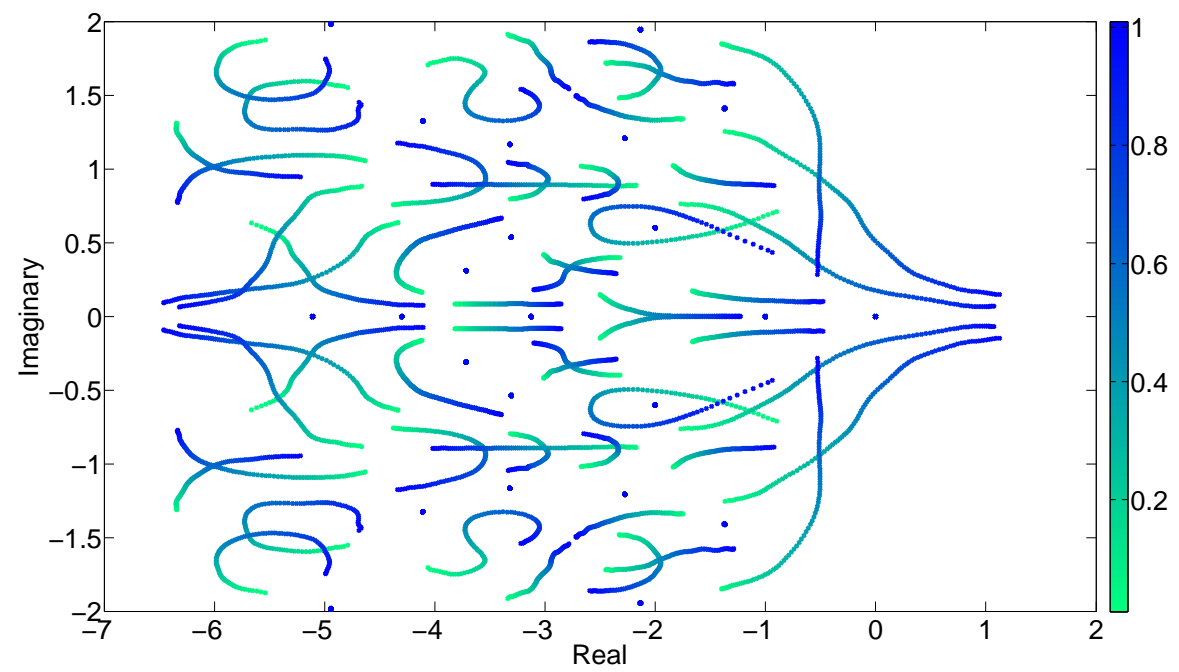

Figure 2. Pole migration of the LPV benchmark system. The value of the scheduling parameter is indicated by colors according to the color-bar on the right.

parameter-dependent. The resulting LPV system can be given as follows:

$$
G_{0}(\rho):\left\{\begin{array}{l}
\dot{x}=T(\rho)^{-1} A_{0}(\rho) T(\rho) x+T(\rho)^{-1} B_{0} u \\
y=C_{0} T(\rho) x+D_{0} u
\end{array}\right.
$$

The third step is evaluating the system over an equidistant grid $\Gamma_{0}$ containing $N_{0}$ parameter values. Then a $d$-th degree polynomial is fitted to each entry of the system matrices in order to deform the eigenvalue trajectories and thus make the model more realistic. Finally, in the fourth step, a gridbased representation is generated by evaluating the model over the grid $\Gamma$. This way, the LPV system is free from any special structure and can be considered as a generic parameter-varying model. To obtain the particular model used in this section the length $N_{0}$ of the initial grid, the length $N$ of the final grid and the degree $d$ of the interpolating polynomials have been chosen to be 60,100 and 14, respectively. Furthermore, to evaluate and challenge the algorithm, a wide range of dynamical behaviors have been covered including:

- parameter varying real- and complex conjugate eigenvalues,

- parameter independent dynamics,

- higher order complex and real eigenvalues (with algebraic multiplicity),

- integrators,

- mixed stability eigenvalues (that cross the imaginary axis),

- complex conjugate - real transitions.

These modes can be recognized on the eigenvalue trajectories depicted in Figure 2. The numerical testing of the algorithm can be performed according to the following steps:

- Eigen-decomposition. Using standard numerical methods, the eigenvalues and the corresponding eigenvectors of the $A_{k}$ matrices are computed at each grid point.

- Identification of integrators. An initial ordering is carried out, based on the absolute value of the eigenvalues in order to locate and label integrators for further computations. The eigenvalues corresponding to the integrators are simply removed from the reduction procedure, and will be added back later, before applying the Procrustes algorithm to smooth the eigenvectors.

- Finding the eigenvalue trajectories. The samples of the pointwise modes over the parameter domain are connected by the Hungarian Algorithm, based on their weighted hyperbolic 
distance. Unstable poles are projected into the unit circle during the construction of the distance matrix, as discussed in APPENDIX A. Continuous eigenvalue trajectories are restored as a function of the scheduling parameter. Integrators are excluded from the pairing and matched individually.

- Detection of multiple eigenvalue trajectories. Eigenvalue trajectories which are in close proximity for every value of the scheduling parameter are grouped together and handled as muliple ones. In a same manner, using the hyperbolic metric between trajectories, irregular behaviours (i.e. complex-real transitions) can be detected and labelled. For example, in this particular example there are two eigenvalues very close to each other, accompanied by occasional complex-real transitions. This represents discontinuity in the eigenspace (assumptions (A) and (B) are violated), hence a numerical correction was necessary. The absolute variance for the corresponding poles over the scheduling parameter domain was found to be in the range of $10^{-7}$ clearly indicating a constant real pole with multiplicity of 2 . Therefore we can replace the values with their average, and the complex eigenvectors can be replaced by the closest real eigenvectors. These steps succesfully resolve the mathematical discontinuity without effecting the input-output behaviour.

- Eigenvector smoothing. The complex Procrustes problem is solved for the matched eigenvectors, where the subspaces of the previously labelled poles are treated accordingly. The smoothness of the modal transformations are analyzed numerically, by comparing the $\dot{\rho}$ dependent terms. For this purpose, a cubic spline interpolation is applied for the eigenvectors obtained from the eigen-decomposition, and for the one obtained through the Procrustes problem. These continuous functions are then evaluated between grid points. The results are summarized in Table I, where the large entries indicate discontinuity in $\partial \bar{T}(\rho) / \partial \rho$, which implies that the state transformation $\bar{T}(\rho)$ is probably not differentiable. The results illustrate the effectiveness of the proposed complex Procrustes smoothing.

\begin{tabular}{l|c|c|c|c} 
& $\max _{\rho} \max \{\cdot\}$ & $\operatorname{mean}_{\rho} \max \{\cdot\}$ & $\max _{\rho}\|\cdot\|_{2}$ & $\operatorname{mean}_{\rho}\|\cdot\|_{2}$ \\
\hline Before Procrustes $\frac{\partial \bar{T}(\rho)}{\partial \rho}$ & 100.52 & 73.6 & 312.28 & 246.28 \\
\hline After Procrustes $\frac{\partial \bar{T}(\rho)}{\partial \rho}$ & 4.57 & 1.07 & 12.6 & 2.85
\end{tabular}

Table I. Numerical comparison of the maximal elements and the matrix norms of modal transformations before- and after smoothing, over the parameter domain. The numerical values were obtained by applying the functions labelling the columns to the matrices labelling the rows, i.e. the 1,1 entry is obtained by computing the maximal entry of $\partial \bar{T}(\rho) / \partial \rho$ as a function of the parameter and maximizing it over $\rho$.

- Modal form. Applying transformation $\bar{T}(\rho)$, the parameter-varying modal form is obtained. At this point a numerical test was carried out to investigate the effect of the $\dot{\rho}$-dependent term. In this particular example this term can be neglected without significant change in the input/output response. (see also Table I).

- Stable-unstable decomposition. By using the continuous eigenvalue trajectories and the parameter-varying modal form, unstable (as well as mixed stability) dynamics can be separated and removed from the system. In the underlying example, five states were separated (see Figure 2) and thus the model reduction is performed for the remaining 75 (stable) states.

- Clustering. Hiearchical clustering of the matched eigenvalue trajectories are performed next, aiming to reveal dynamical redundancies of the system. Figure 3 shows the corresponding dendrogram plot, which is used for representing the arrangement of the clusters. In Figure 3 the height of each line equals to the distance between the two data objects (either eigenvalue trajectory or cluster of trajectories, computed by (9) ) below. Based on the dendrogram, different number of clusters can be generated and it is the task of the user to decide. To make this decision the following trade-off has to be taken into consideration: large number of clusters corresponds to smaller sized groups, in which state elimination is generally more conservative (consider the extreme case, where each cluster contains a single mode). On the other hand, small number of clusters imply higher dimensional groups with increasing numerical burden (on the other extreme: the entire system considered as a single cluster). 
Taking this into account, the given system are subdivided into five clusters (see Figure 3), with the following dimensions: 26 (red), 34 (purple), 7 (blue), 6 (green) and 2 (black). Note that the last cluster cannot be reduced, since it contains a single complex-conjugate mode. According to Figure 3, a dynamic dissimilarity can be observed, implying the preservation of this mode in question.

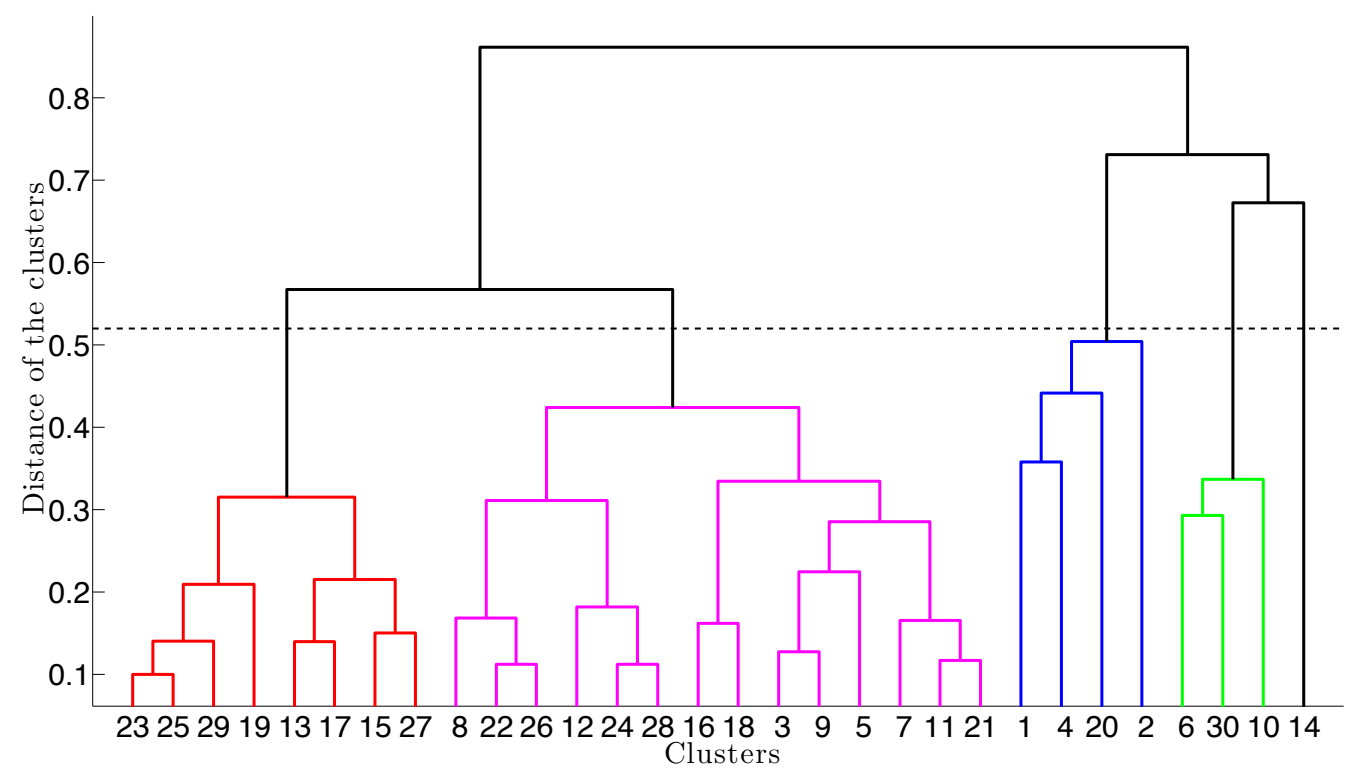

Figure 3. Dendrogram for clustering in the benchmark example. The horizontal axis is labelled by the indices of the data objects (eigenvalue trajectories) while the vertical axis shows the similarity distance computed by using (9). The dashed line indicates the threshold where the dendrogram was cut to obtain the 5 clusters.

In addition, the cophenetic correlation coefficient is computed, which is often used for characterizing a dendrogram: how faithfully does it represent the similarities among data objects [40]. The magnitude of the cophenetic correlation should be close to 1 for the case of a good description. The computed value was 0.83 , which shows that the selected hyperbolic metric is indeed a good indicator for comparing different dynamical behaviours.

- Computing Gramians. The parameter-dependent controllability and observability Gramians are computed for each individual cluster. By fixing the structures of the Gramians as $X_{c}^{(\ell)}(\rho)=X_{c, 0}^{(\ell)}+\rho X_{c, 1}^{(\ell)}$ and $X_{o}^{(\ell)}(\rho)=X_{o, 0}^{(\ell)}+\rho X_{o, 1}^{(\ell)}$ the iterative optimization (Section 4.2) is carried out for each sub-system by using the MOSEK optimization tool [42]. This corresponds to $(351+595+28+21) \times 4$ decision variables in four separate optimization problems, which is a significant decrease compared to the $5550 \times 4$ variables involved in the single LMI problem for the entire 75 dimensional system.

- Singular Value Decomposition. In order to determine the number of states with the largest contributions to the input-output behaviour of each subsystem, the parameter-varying generalized singular values are computed for the subsystems by [9] The number of most significant singular values followed by the dimension of the corresponding cluster are $5 / 26$, $5 / 34,3 / 7$ and $2 / 6$.

- Balanced Transformation. The parameter-varying balancing transformations and their inverses can be computed for each subsystem, using the singular value decomposition [9]. Applying these transformations, balanced forms are obtained, resulting in a $\rho, \dot{\rho}$ dependent system. The balanced models are then truncated according to the most significant singular values, computed in the previous step. That is, the 75 dimensional stable part is reduced to 17 dimension.

- Reduced model construction. Finally, the individually reduced subsystems are joined together and the five dimensional unstable dynamics are added back. Hence the final, reduced-order 


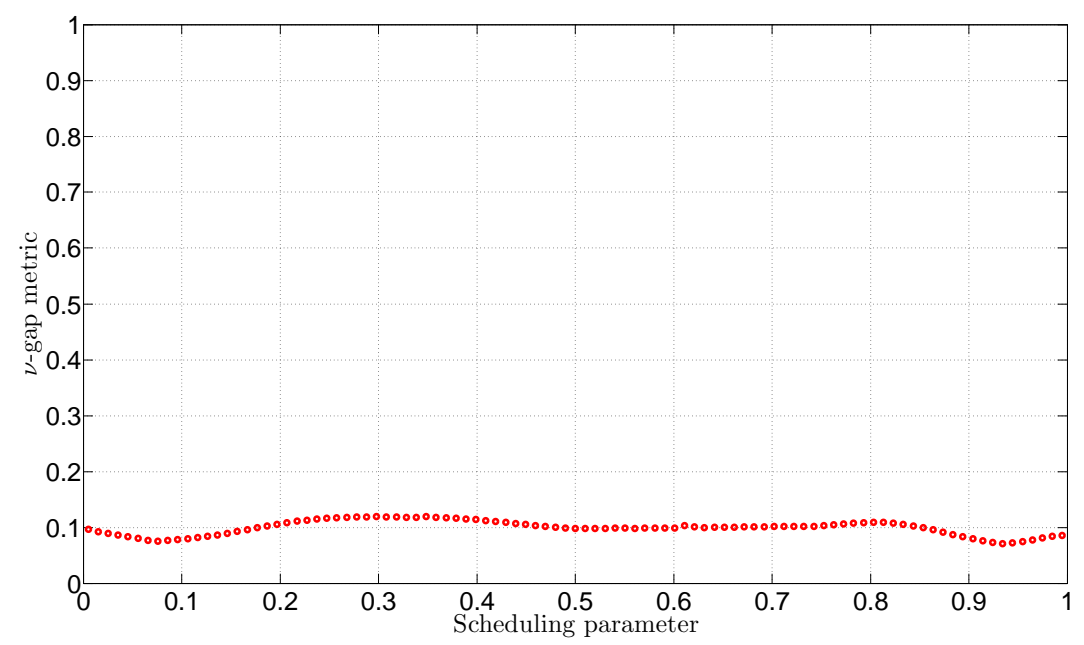

Figure 4. $\nu$-gap metric of the full (80) and reduced (22) order model over the scheduling parameter domain

LPV model has 22 states (compared to the original 80 ) and given in a grid-based fashion, depending on $\rho$ and $\dot{\rho}$.

- Evaluation of the reduced model. In order to validate the reduced model, various numerical properties have been investigated. One of the main motivation of model reduction is to obtain a smaller dimensional representation, which is suitable for controller design. From this point of view, similartity of closed-loop behaviours between the high-order and low-order models is of capital importance. In order to take the feedback control objective into account, the widelyused $\nu$-gap metric is evaluated, defined between LTI systems, $G_{1}(j \omega)$ and $G_{2}(j \omega)$, as [43], [44]:

$$
\begin{aligned}
& \delta_{\nu}\left(G_{1}(j \omega), G_{2}(j \omega)\right)= \\
& \left\|\left(I+G_{2}(j \omega) G_{2}^{*}(j \omega)\right)^{-\frac{1}{2}}\left(G_{1}(j \omega)-G_{2}(j \omega)\right)\left(I+G_{1}^{*}(j \omega) G_{1}(j \omega)\right)^{-\frac{1}{2}}\right\|_{\infty} .
\end{aligned}
$$

Essentially, if $\delta_{\nu}\left(G_{1}, G_{2}\right) \leq \beta$, then a controller, which stabilizes $G_{2}$ also stabilizes $G_{1}$, with a stability margin of $\beta$ [44]. For identical systems $\delta_{\nu}\left(G_{1}, G_{2}\right)=0$, otherwise it is $0 \leq \delta_{\nu} \leq 1$. For LPV systems (15) can be interpreted in different ways. One possibility is to compare point-wise LTI systems of the LPV dynamics by taking:

$$
\max _{\omega} \delta_{\nu}\left(G_{k}(j \omega), \tilde{G}_{k}(j \omega)\right)
$$

at each grid point $k \in[1100]$. Figure 4 shows the evaluation of the above expression for models interpolated between the original grid points. The maximal value is 0.12 , implying a satisfactory similarity between the full and reduced order models. The second representation of (15) is illustrated on Figure 5, where the interpolated systems are compared at every $\omega_{i}$ frequency, i.e.:

$$
\max _{\rho} \delta_{\nu}\left(G(\rho)\left(j \omega_{i}\right), \tilde{G}(\rho)\left(j \omega_{i}\right)\right)
$$

Figure 5 also provides an insight on the frequency-domain properties of the LPV model reduction.

Finally, the proposed algorithm is compared with a local reduction approach. At each grid point a balanced transformation based model reduction is performed for the corresponding high dimensional LTI model. The local Hankel singular values imply lower dimensional models, within the range of $2-10$, for the sake of consistency the dimension has been 


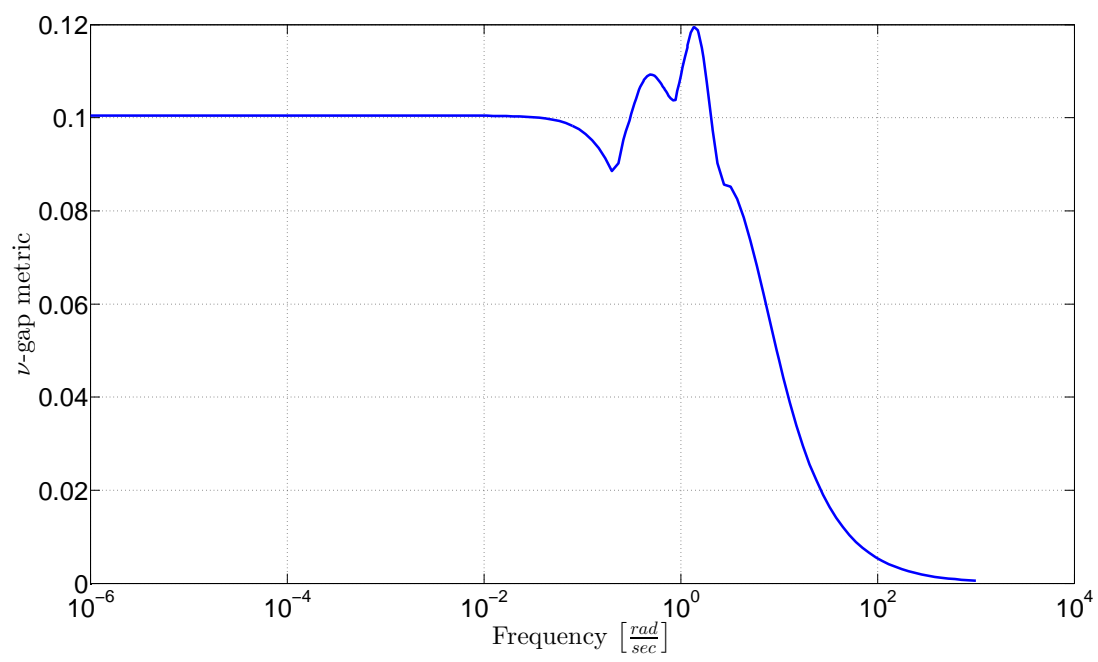

Figure 5. Frequency-wise maximum $\nu$-gap metric of the full (80) and reduced (22) order model over the scheduling parameter domain
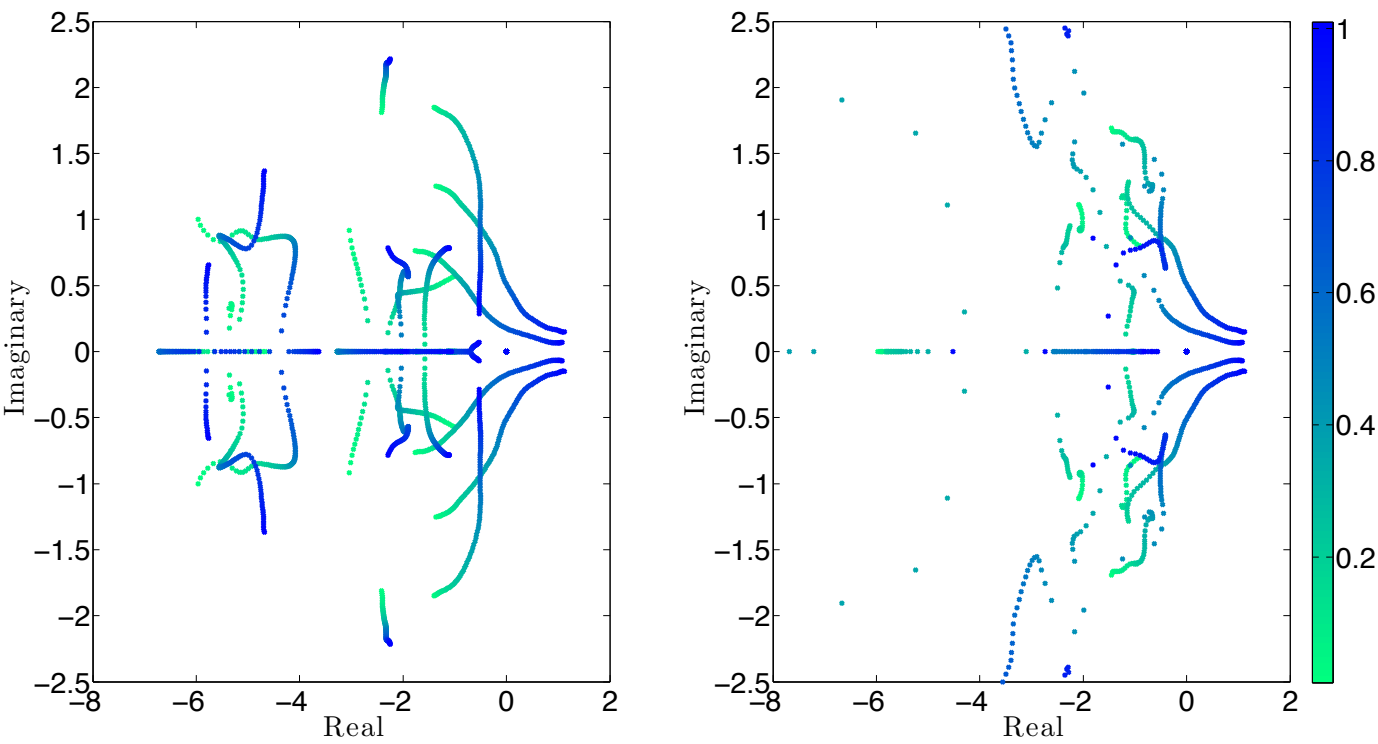

Figure 6. Pole migration map for the reduced order model (left) and for the collection of locally reduced systems (right). The value of the scheduling parameter is indicated by colors according to the color-bar on the right.

fixed for 10 at each grid-point. This fact indicates some conservativeness of the proposed methodology, which, in general preserves more states as a consequence of the modal decomposition. The pole migration maps for the two approaches are compared in Figure 6. It can be observed, that the resulting parameter varying model has a smooth pole map, which makes the LPV model generation straightforward and less challenging. On the other hand, the pole map for the set of locally reduced models shows large variation (see the right plot in Figure 6). This implies the need of a more refined interpolation technique to successfully recover time domain behaviour of the original plant, which also makes control design virtually infeasible.

The complete flowchart representing the model reduction workflow is presented in Fig. 7. 


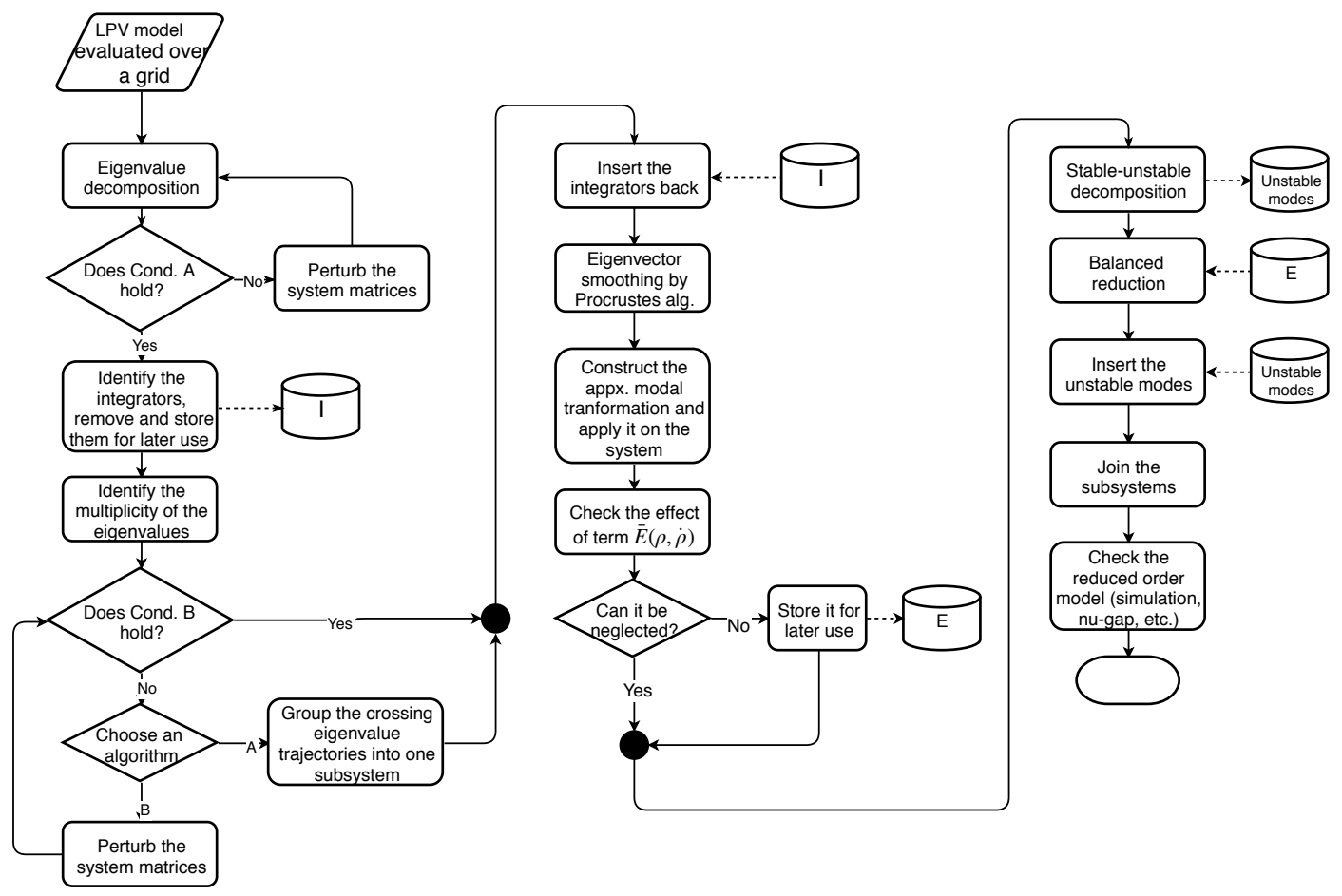

Figure 7. The complete flowchart of the model reduction algorithm.

\subsection{B-1 aircraft}

Our second example is the publicly available nonlinear aeroelastic model of the Rockwell B-1 aircraft. This model is widely known and investigated due to the aeroelastic effects observed at subsonic speeds. To obtain an LPV system for model reduction the simulation model from [45] is used. By linearizing the nonlinear model at the flight altitude of $15000 \mathrm{ft}$ with different Mach numbers between $0.6-0.75$, a grid-based LPV dynamics with 20 state variables are obtained. The 20 states consist of the 10 dimensional rigid body dynamics (bank angle $\phi$, pitch angle $\theta$, yaw angle $\psi$, roll, pitch and yaw rates of the center of gravity: $p, q, r$, and $x-y-z$ axis velocities in the body coordinate frame: $U, V, W$ and altitude $h$ ) and the additional 10 states of the five flexible modes. Eight measured outputs are considered: flight path angle $\gamma$, the roll, pitch and yaw rates of the center of gravity, and the lateral and vertical accelerations of the cockpit and center of gravity. The model has 9 inputs: throttle $T$, right and left (symmetric) horizontal tails: $\delta_{H R}, \delta_{H L}$, upper and lower split rudder surfaces: $\delta_{R U}, \delta_{R L}$, wing upper-surface spoilers: $\delta_{S R}, \delta_{S L}$ and canard control vanes: $\delta_{C V R}, \delta_{C V L}$. For these actuators a 13 dimensional LTI dynamics is given and used through the augmentation of the LPV model. Accordingly, the final model used for testing the model reduction algorithm is 33 dimensional.

The reduced order model can be obtained by performing the following steps. After obtaining the parameter-varying modal form, three mixed stability modes are removed. The resulting 30 states can be grouped into 3 clusters with dimensions of 23,5 and 2. Based on the parameter-varying singular values 7,5 and 2 significant dimensions can be identified, i.e. only the largest block has to be reduced. Hence, the resulting reduced-order model has a 14 dimensional stable and 3 dimensional unstable part.

To investigate the numerical properties of the reduced order model, time-domain simulations are carried out first. In these simulations the flight speed is set to vary as:

$$
V(t)=0.675+0.05 \sin (0.2 t)
$$




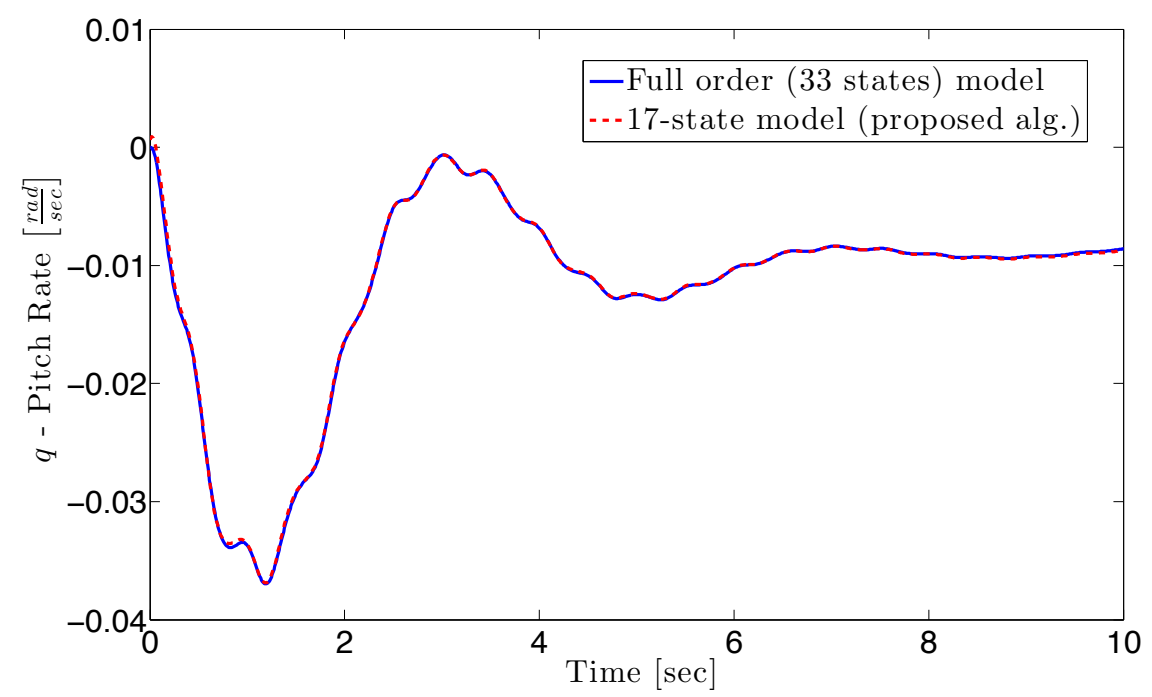

Figure 8. Pitch rate response to a positive one degree deflection of the symmetric horizontal tail.

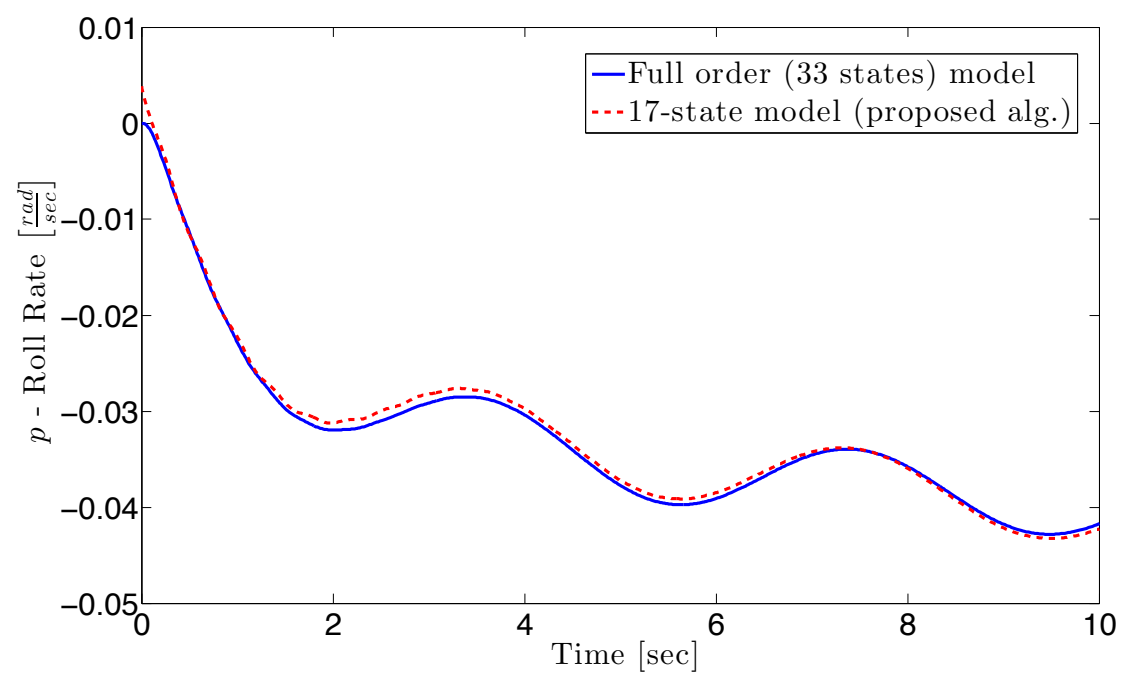

Figure 9. Roll rate response to a one degree asymmetric horizontal tail deflection.

which corresponds to a maximal acceleration of $\approx 3.3 \frac{\mathrm{m}}{\mathrm{sec}^{2}}$. Figure 8 compares the pitch rate responses to a positive-one-degree commanded symmetric-horizontal-tail deflection of the full order model (with 33 states) and the reduced order one (17 states). It can be depicted that the two models give almost identical responses. Figure 9 shows the roll rate responses, where the horizontal-tail deflections are set asymmetrically $\delta_{H L}=-1^{\circ}, \delta_{H R}=1^{\circ}$. Again, only a small discrepency can be observed between the two models.

As noted previously, the LPV balanced transformation and reduction suffers from the curse of dimensionality, i.e. it runs into numerical problems for higher dimensional systems. Accordingly, it cannot be applied directly to our first benchmark example in Section 5.1. At the same time, for the case of the 33 dimensional model it is possible to solve the underlying LMI problems and consequently obtain a reduced description. Accordingly, after a stable-unstable decomposition the parameter-varying singular values have been computed for the stable part by using the parametervarying Gramians and the corresponding balancing transformation [9]. The singular-value plots are given in Figure 10 implying the elimination of 17 states, which corresponds to a 16 dimensional 


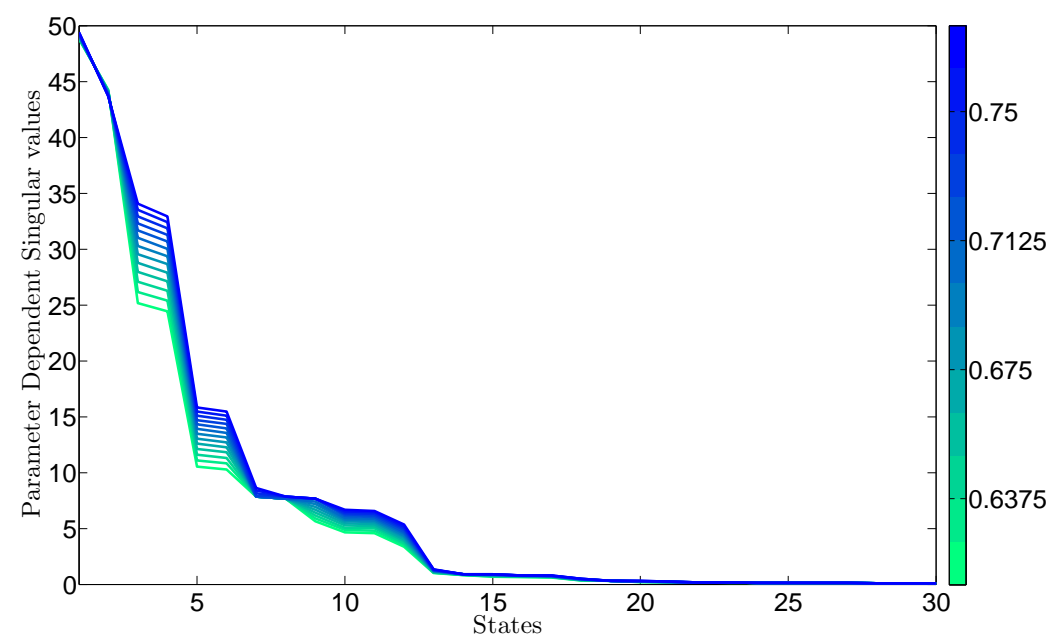

Figure 10. Singular Values for the stable part of the B1 aircraft model. The value of the scheduling parameter is indicated by colors according to the color-bar on the right.
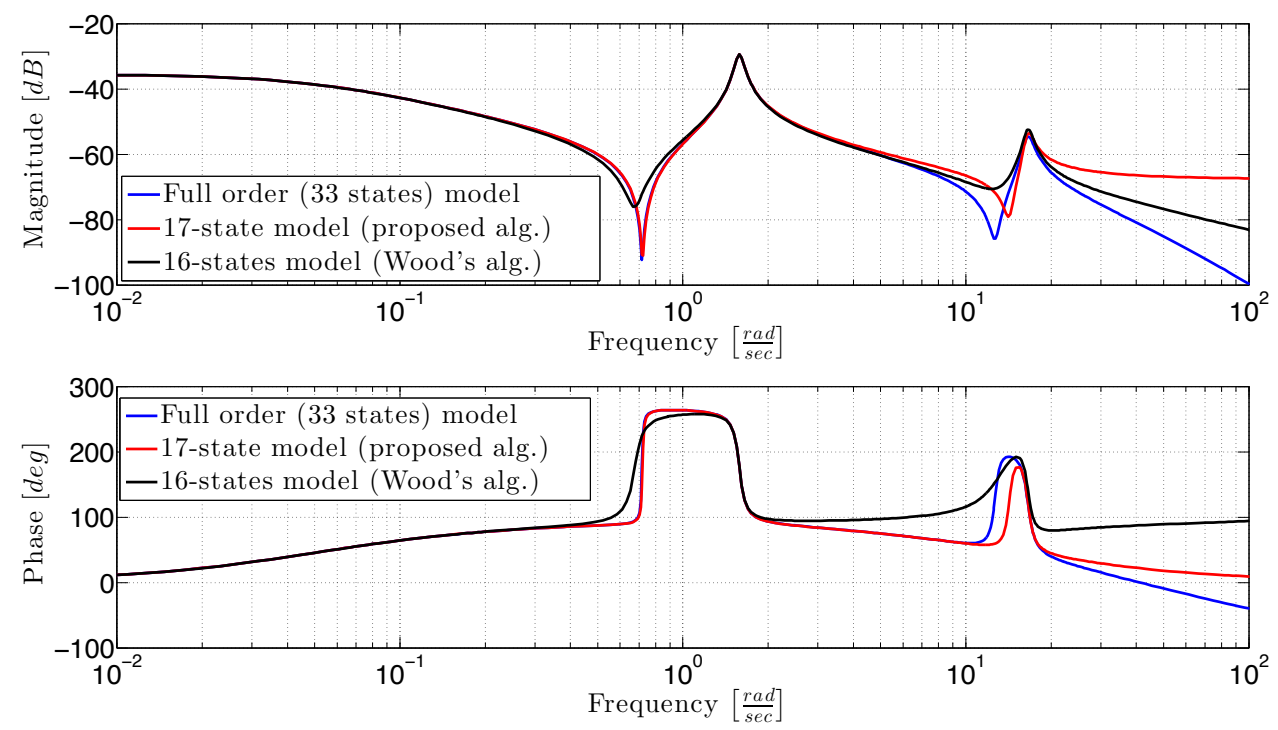

Figure 11. Bode plots from $\delta_{R U}$ to $r$ of the full model (33 states) at 0.6 Mach compared to the two reduced order models. The 17-state model has been obtained by the proposed algorithm, while the balanced reduction algorithm from [9] has generated the 16-state model.

reduced system. This shows and verifies that the proposed approach indeed give a good estimation for the order of the reduced system.

Finally, the frequency-domain properties are compared in Figures 11 and 12. The Bode amplitude and phase diagrams of the i) full (blue), ii) reduced with the proposed approach (red) and iii) reduced by the method of [9] (black), models have been compared from the upper rudder surface $\delta_{R U}$ to the roll rate $r$ at two different flight speed $(0.6 M$ and the interpolated point $0.7312 M)$. Note that, according to the applied balanced residualization, model mismatch appears in the higher frequency domain. Still, the frequency domain approximation is satisfactory in the operation domain. 

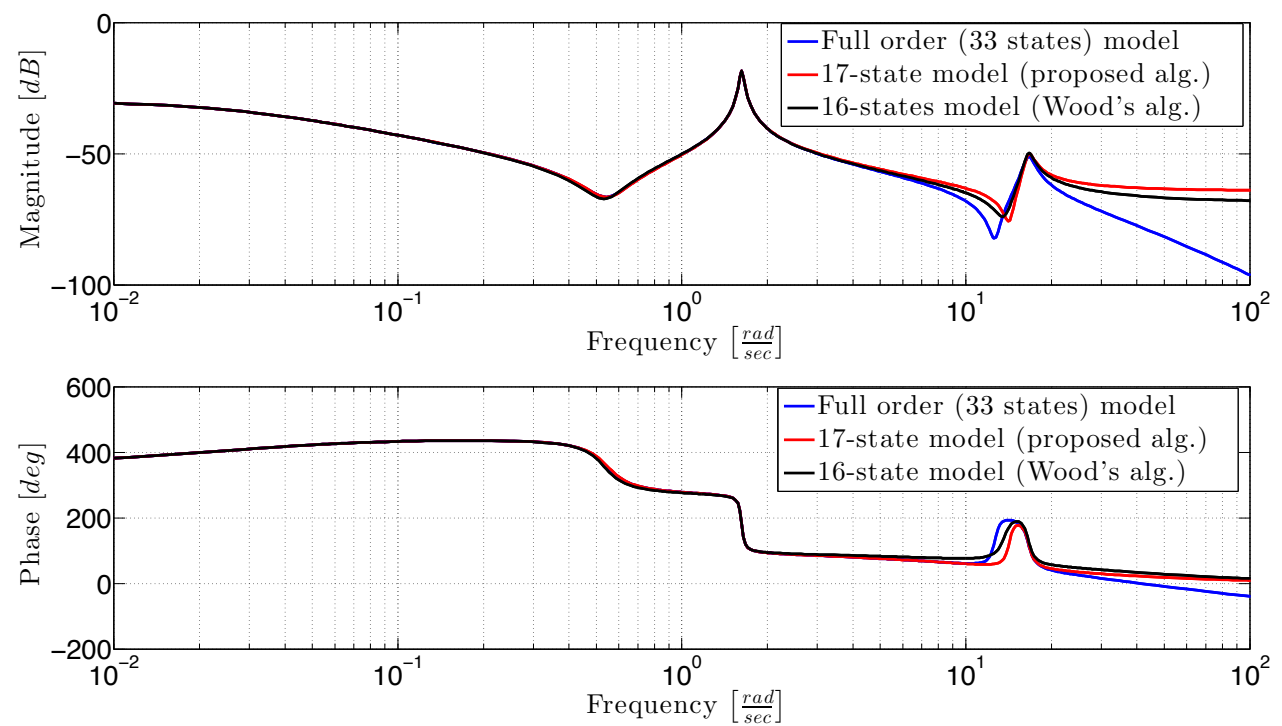

Figure 12. Bode plots from $\delta_{R U}$ to $r$ of the full model (33 states) at 0.7312 Mach compared to the two reduced order models. The 17 -state model has been obtained by the proposed algorithm, while the balanced reduction algorithm from [9] has generated the 16-state model.

\section{CONCLUSION}

A novel model order reduction algorithm, based on approximate system decomposition, has been developed for LPV systems. The fundamental element of the algorithm is a parameter varying state transformation, which transforms (at least approximately) the LPV system into modal form. For this purpose the local transformation matrices have been appropriately modified and interpolated. The obtained structure is then subjected to a dynamic similarity analysis by using a hierarchical clustering method. This step reveals dynamically redundant modes of the system, which are grouped together to form parameter-varying subsystems. Finally, these smaller dimensional subsystems are separately reduced by parameter varying balanced reduction algorithm. The effectiveness of the methodology is illustrated by in-depth numerical studies.

The present framework requires some assumptions on the LPV system to be reduced. It is shown that most of these assumptions can be relaxed if slight modifications are allowed on the system matrices. The most stringent constraint is the one limiting the dimension of the scheduling variable to 1 . Though the main concept of the proposed model reduction method can be extended to systems having more than one scheduling parameters, the numerical details of the algorithm have to be elaborated. The most critical point here is the separation of the modal subsystems, i.e. the generalization of the Hungarian algorithm and the iterative Procrustes smoothing. But once this is done the procedure can be continued since the clustering and the balanced reduction do not depend on the dimension of the scheduling parameter. The extension of the modal decomposition to multiparameter LPV systems is one important direction for the future research.

Since LTI and parametric LTI systems are special classes of LPV systems, so the proposed method remains applicable, though it may be not the most efficient approach, in those special cases as well. If the system to be reduced is LTI, then there are no parameter dependent eigenvalues/eigenvectors so the modal transformation and also the clustering can be omitted and the algorithm boils down to a simple balanced reduction. In parametric LTI case the time derivative of the parameter is zero, so the $\dot{\rho}$-dependent terms causing most of the difficulties in model reduction do not appear in the transformed models.

The well-known numerical problems related to eigen-decomposition is certainly one area which also needs further considerations, especially for very large dimensional and ill-conditioned 
problems. Furthermore, currently we are seeking more rigoruous methods for the decomposition of LPV systems. Guaranteed error bounds of the method will also be part of our future research.

\section{ACKNOWLEDGEMENT}

The research leading to these results is part of the FLEXOP project. This project has received funding from the European Unions Horizon 2020 research and innovation programme under grant agreement No 636307. This paper was also supported by the János Bolyai Research Scholarship of the Hungarian Academy of Sciences. The authors would like to thank Peter Seiler (University of Minnesota) and Arnar Hjartarson (formerly MUSYN Inc.) for providing insight related to the B-1 simulator model, originally developed by David Schmidt. The authors also would like to thank prof. Gary Balas (University of Minnesota) for initiating the research on control oriented modeling of flexible aircrafts, which gave motivation for working on large-scale LPV systems.

\section{A. HYPERBOLIC DISTANCE}

This section introduces the hyperbolic distance metric as a possible measure of dynamic similarity between two poles of LTI systems. The derivation is based on the fundamental results of system theory, which relate the location of the dominant poles to the transient response. This property is more pronounced in a discrete time setup, therefore all the stable eigenvalues are mapped into the unit circle for further discussion. Each $z^{\prime} \in \mathbb{C}$ discrete-time eigenvalue defines a first-order SISO transfer function $G^{\prime}(z)$ with a single pole located at $z^{\prime}$ :

$$
G^{\prime}(z)=\frac{a^{\prime}(z)}{z^{\prime}-z}
$$

where $a^{\prime}(z)$ is an arbitrary polynomial in $z$. The collection of all possible transfer functions, generated by a single eigenvalue $z^{\prime}$ is denoted by $\mathfrak{G}\left[z^{\prime}\right]$. It is clear, that $\mathfrak{G}\left[z^{\prime}\right] \subset H^{2}(\mathbb{D})$, where $H^{2}(\mathbb{D})$ denotes the Hardy space of complex-valued functions [46]. This is an infinite dimensional vector space. Among its possible orthonormal bases the discrete Laguerre system has received distinct attention in system and control theory [47]. The Laguerre system is generated by any complex number $z^{\prime \prime}$ as follows:

$$
\Phi_{m}^{\prime \prime}(z)=\frac{\sqrt{1-\left|z^{\prime \prime}\right|^{2}}}{z-z^{\prime \prime}}\left(\frac{1-\left(z^{\prime \prime}\right)^{*} z}{z-z^{\prime \prime}}\right)^{m} \quad(m=0,1,2 \ldots),
$$

Using the Laguerre system as a basis, each transfer function in $\mathfrak{G}\left[z^{\prime}\right]$ can be represented through the linear combination of a $\Phi_{m}(z)$, i.e.

$$
G^{\prime}(z)=\sum_{m=0}^{\infty} l_{m} \Phi_{m}^{\prime \prime}(z)
$$

where $l_{m}$ are the corresponding linear coefficients. It follows from the results in [48] and [49] that under the given conditions, the ratio of two consecutive coefficients in (21) is always constant and depends only on the generator elements $z^{\prime \prime}$ and the $z^{\prime}$. That is: $l_{m+1} / l_{m}=r$ for all $m=\{1,2, \ldots\}$ and $r$ is called the convergence factor, because it characterizes the convergence of the Laguerre series (21). Small coefficient implies that only a few elements are dominant in (21), i.e. few basis functions are enough to capture the dynamic behaviour of $G^{\prime}(z)$. The metric $r$ is thus characterizes the similarity between the transfer functions $G^{\prime}(z)$ generated by $z^{\prime}$ and the basis elements parameterized by $z^{\prime \prime}$. Therefore, it can be considered as a possible measure of the dynamic similarity between $z^{\prime}$ and $z^{\prime \prime}$. Furthermore, it can be proved (see e.g. [48]) that $r$ can be computed by the following simple formula

$$
r=h\left(z^{\prime}, z^{\prime \prime}\right)=\left|\frac{z^{\prime}-z^{\prime \prime}}{1-z^{\prime *} z^{\prime \prime}}\right|
$$

which is also known as the pseudo-hyperbolic metric between $z^{\prime}$ and $z^{\prime \prime}$ [50], [51].

Being defined on the unit disk, this metric can only be applied on stable, discrete eigenvalues. In order to compare continuous eigenvalues, they have to be "discretized" first by the formula $\lambda_{d}=\exp \left(\lambda_{c} T_{s}\right)$, where $T_{s}$ is a sufficiently small sampling time and $\lambda_{c}, \lambda_{d}$ denote the continuous eigenvalue and its discrete counterpart, respectively. Formula (22) can be applied to discrete, unstable eigenvalues as well provided that they are transformed into the unit disk by the mapping $f(z)=1 / z^{*}$. (Note that $f(z)$ reflects the unstable eigenvalue across the unit circle, which guarantees that the distance between a stable and a (transformed) 
unstable eigenvalue is small if they are close to each other in the sense that they can be considered as two consecutive points of the same eigenvalue trajectory. This property is important when mixed stability eigenvalues have to be identified.) For notational convenience, the hyperbolic distance of two stable/unstable continuous/discrete eigenvalues will also be denoted by $h(\cdot, \cdot)$ and the transformations necessary to use (22) are assumed to be performed beforehand.

\section{REFERENCES}

1. Peni T, Seiler P. Computation of lower bounds for the induced $\mathcal{L}_{2}$-norm of LPV systems. International Journal of Robust and Nonlinear Control 2016; 26(4):646-661.

2. Bokor J, Balas G. Detection filter design for LPV systems-a geometric approach. Automatica 2004; 40:511-518.

3. Pfifer H, Seiler P. Robustness analysis of linear parameter varying systems using integral quadratic constraints. International Journal of Robust and Nonlinear Control 2015; 25(15):2843-2864.

4. Wu F. Control of linear parameter varying systems. PhD Thesis, University of California at Berkeley 1995.

5. Moreno CP, Seiler PJ, Balas GJ. Model Reduction for Aeroservoelastic Systems. Journal of Aircraft 2014; 51(1):280-290.

6. Kwiatkowski A, Werner H. PCA-Based Parameter Set Mappings for LPV Models With Fewer Parameters and Less Overbounding. IEEE Transactions on Control Systems Technology 2008; 16(4):781-788.

7. Rizvi SZ, Mohammadpour J, Toth R, Meskin N. A Kernel-Based PCA Approach to Model Reduction of Linear Parameter-Varying Systems. IEEE Transactions on Control Systems Technology 2016; 24(5):1883-1891.

8. Antoulas A. Approximation of Large-Scale Dynamical Systems. Advances in Design and Control, SIAM, 2005.

9. Wood GD. Control of Parameter-Dependent Mechanical Systems. PhD Thesis, University of Cambridge 1995.

10. Wood GD, Goddard PJ, Glover K. Approximation of linear parameter-varying systems. Conference on Decision and Control (CDC), 1996; 406-411.

11. Moore BC. Principle Component Aanalysis in Linear Systems: Controllability, Observability, and Model Reduction. IEEE Transactions on Automatic Control 1981; 26(1):17-32.

12. Theis J, Seiler P, Werner H. Model Order Reduction by Parameter-Varying Oblique Projection. American Control Conference 2016;

13. Poussot-Vassal C, Roos C. Generation of a reduced-order LPV/LFT model from a set of large-scale MIMO LTI flexible aircraft models. Control Engineering Practice 2012; 20:919-930.

14. Theis J, Takarics B, Pfifer H, Balas GJ, Werner H. Modal matching for LPV model reduction of aeroservoelastic vehicles. AIAA Science and Technology Forum, 2015.

15. Adegas F, Sonderby I, Hansen M, Stoustrup J. Reduced-order LPV model of flexible wind turbines from high fidelity aeroelastic codes. IEEE Conference on Control Applications, 2013; 424-429.

16. de Caigny J, Pintelon R, Camino JF, Swevers J. Interpolated Modeling of LPV Systems. IEEE Transactions on Control Systems Technology 2014; 22(6):2232-2246.

17. Panzer H, Mohring J, Eid R, Lohmann B. Parametric Model Reduction by Matrix Interpolation. Automatisierungstechnik 2010; 58:475-484.

18. Geuss M, Panzer H, Lohmann B. On parametric model order reduction by matrix interpolation. European Control Conference, 2013; 3433-3438.

19. Benner P, Grundel S, Hornung N. Parametric model reduction with small $\mathcal{H}_{2}$-error using radial basis functions. Advances in Computational Mathematics 2015; 41:1231-1253.

20. Grabner N, Mehrmann V, Quraishi S, Schroder C, Wagner U. Numerical methods for parametric model reduction in the simulation of disk brake squeal. ZAMM - Journal of Applied Mathematics and Mechanics 2016; 96(12):13881405

21. Amsallem D, Cortial J, Carlberg K, Farhat C. A method for interpolating on manifolds structural dynamics reducedorder models. International Journal for Numerical Methods in Engineering 2009; 0:1-16.

22. Amsallem D, Farhat C. An online method for interpolating linear parametric reduced-order models. SIAM Journal on Scientific Computing 2011; 33(5):2169-2198.

23. Alkhoury Z, Petreczky M, Mercére G. Comparing global input-output behavior of frozen-equivalent LPV statespace models. arXiv:1703.03679 2017;

24. Amsallem D, Farhat C, Haasdonk B ( (eds.)). Special Issue: Model Reduction. International Journal for Numerical Methods in Engineering, 2015.

25. Varga A. Enhanced Modal Approach for Model Reduction. Mathematical Modelling of Systems 1995; 1:91-105.

26. Gőzse I, Luspay T, Péni T, Szabó Z, Vanek B. Model order reduction of LPV systems based on parameter varying modal decomposition. Conference on Decision and Control (CDC), 2016; 7459-7464.

27. Lipták G, Luspay T, Péni T, Takarics B, Vanek B. LPV model reduction methods for aeroelastic structures. Accepted to IFAC World Congress, 2017.

28. Patartics B, Luspay T, Péni T, Takarics B, Vanek B, Kier T. Parameter varying flutter suppression control for the BAH jet transport wing. Accepted to IFAC World Congress, 2017.

29. Marcos A, Balas G. Development of linear-parameter-varying models for aircraft. Journal of Guidance, Control and Dynamics 2004; 27(2):218-228.

30. Ewins DJ. Modal Testing: Theory and Practice. John Wiley \& Sons, 1984

31. Burkard R, Dell'Amico M, Martello S. Assignment Problems. SIAM, 2009.

32. Lovasz L, Plummer MD. Matching Theory, Annals of Discrete Mathematics, vol. 29. North-Holland Mathematics Studies, 1986.

33. Golub GH, Loan CFV. Matrix computations. Johns Hopkins University Press, 2013.

34. Lancaster P. On eigenvalues of matrices dependent on a parameter. Numerische Mathematik 1964; 6:377-387. 
35. Juang JN, Ghaemmaghami P, Lim KB. Eigenvalue and eigenvector derivatives of a nondefective matrix. Journal of Guidance, Control and Dynamics 1989; 12(4):480-486.

36. Lancaster P, Markus AS, Zhou F. Perturbation Theory for Analytic Matrix Functions: The Semisimple Case. SIAM Journal on Matrix Analysis and Applications 2003; 25(3):606-626.

37. Dieci L, Papini A. Continuation of eigendecompositions. Future Generation Computer Systems 2003; 19:11251137.

38. Quian J, Chu D, Tan RCE. Analyticity of Semisimple Eigenvalues and Corresponding Eigenvectors of MatrixValued Functions. SIAM Journal on Matrix Analysis and Applications 2015; 36(4):1542-1566.

39. Dieci L, Papini A, Pugliese A, Spadoni A. Current Challenges in Stability Issues for Numerical Differential Equations, chap. Continuous Decompositions and Coalescing Eigenvalues for Matrices Depending on Parameters. Lecture Notes in Mathematics 2082, CIME Foundation Subseries, Springer, 2011; 173-264.

40. Manning CD, abd H Schütze PR. Introduction to Information Retrieval. Cambridge University Press, 2008.

41. Hastie T, Tibshirani R, Friedman J. The Elements of Statistical Learning. Data Mining, Inference, and Prediction, Springer, 2009.

42. ApS M. The MOSEK optimization toolbox for MATLAB manual. Version 7.1 (Revision 28) edn. 2015. URL http://docs.mosek.com/7.1/toolbox/index.html.

43. Georgiou TT. On the computation of the gap metric. Systems Control Letters 1988; 11:253-257.

44. Vinnicombe G. Measuring robustness of feedback systems. PhD Thesis, Department of Engineering, University of Cambridge 1993.

45. Schmidt D. A nonlinear simulink simulation of a large flexible aircraft. Technical Report, MUSYN Inc. and NASA Dryden Flight Research Center 2013.

46. Toth R. Modeling and Identification of Linear Parameter-Varying Systems. Springer-Verlag, 2010.

47. Soumelidis A, Schipp F, Bokor J. Pole Structure Estimation from Laguerre Representations Using Hyperbolic Metrics on the Unit Disc. Conference on Decision and Control (CDC) and European Control Conference, 2011; 2136-2141.

48. Heuberger PSC, den Hof PMJV. Modelling and Identification with Rational Orthogonal Basis Functions. Springer: Dordrecht, 2006.

49. Soumelidis A, Bokor J, Schipp F. Realizing system poles identification on the unit disc based on Laguerre representations and hyperbolic metrics. Mediterranean Conference on Control and Automation (MED), 2013; $1208-1213$.

50. Beardon A, Minda D. The hyperbolic metric and geometric function theory. Proceedings of the International Workshop on Quasiconformal Mappings and their Applications, 2000.

51. Szabó Z, Bokor J. Non-Euclidean Geometries in Modeling and Control. Széchenyi University Press, 2015. 